\title{
Stem Cell-Derived Exosomes: a New Strategy of Neurodegenerative Disease Treatment
}

\author{
Nashmin Fayazi ${ }^{1}$ - Mohsen Sheykhhasan ${ }^{1} \cdot$ Sara Soleimani AsI ${ }^{2} \cdot$ Rezvan Najafi $^{1}$ (D) \\ Received: 26 September 2020 / Accepted: 5 February 2021 / Published online: 21 March 2021 \\ (C) The Author(s), under exclusive licence to Springer Science+Business Media, LLC, part of Springer Nature 2021
}

\begin{abstract}
Short-term symptomatic treatment and dose-dependent side effects of pharmacological treatment for neurodegenerative diseases have forced the medical community to seek an effective treatment for this serious global health threat. Therapeutic potential of stem cell for treatment of neurodegenerative disorders was identified in 1980 when fetal nerve tissue was used to treat Parkinson's disease (PD). Then, extensive studies have been conducted to develop this treatment strategy for neurological disease therapy. Today, stem cells and their secretion are well-known as a therapeutic environment for the treatment of neurodegenerative diseases. This new paradigm has demonstrated special characteristics related to this treatment, including neuroprotective and neurodegeneration, remyelination, reduction of neural inflammation, and recovery of function after induced injury. However, the exact mechanism of stem cells in repairing nerve damage is not yet clear; exosomes derived from them, an important part of their secretion, are introduced as responsible for an important part of such effects. Numerous studies over the past few decades have evaluated the therapeutic potential of exosomes in the treatment of various neurological diseases. In this review, after recalling the features and therapeutic history, we will discuss the latest stem cell-derived exosome-based therapies for these diseases.
\end{abstract}

Keywords Stem cells $\cdot$ Exosome $\cdot$ Neurodegenerative disease $\cdot$ Treatment

\section{Introduction}

Gradual loss of neuronal structure and function in both the central and peripheral nervous system leads to a heterogeneous group of disorders which are called neurodegenerative diseases like Alzheimer disease (AD), Parkinson disease (PD), Huntington's disease (HD), multiple sclerosis (MS), and amyotrophic lateral sclerosis (ALS) [1]. Aging is known as the most important risk factor for many neurological diseases [2]. As the world's older population is growing, the number of people with degenerative nerve diseases will overtake cancer in the near future and become the second leading cause of death in the world [3]. Neurodegenerative diseases impact a wide range of individual activities such as balance,

Nashmin Fayazi and Mohsen Sheykhhasan contributed equally to this work.

Rezvan Najafi

re.najafi@umsha.ac.ir; najafi2535@gmail.com

1 Research Center for Molecular Medicine, Hamadan University of Medical Sciences, Hamadan, Iran

2 Anatomy Department, School of Medicine, Hamadan University of Medical Sciences, Hamadan, Iran movement, memory, speech, and breathing [4]. Although the cause of many of these diseases is not yet known, a combination of genetic factors and environmental conditions may play a role in causing these diseases [4]. Death of neurons is the most common feature of these diseases that affected different areas of the brain such as cortical and hippocampal regions in $\mathrm{AD}$ and striatal regions in PD [5]. Depending on the area of the brain which is affected, these diseases can show mild or severe symptoms [6]. Due to the relatively long duration of these diseases and their high cost of treatment, these diseases have become a serious challenge for both patients and community healthcare, so the international community urgently needs to find an effective treatment for this global problem.

Neurodegenerative diseases are multifactorial disorders [7]. The multicommon pathogenic mechanisms involved in neurological diseases including aggregation and degradation of defective protein and formation of inclusion bodies, dysregulation of intracellular protein trafficking and endocytic sorting, mitochondrial dysfunction, oxidative stress and generation of reactive oxygen species (ROS), iron accumulation, inflammation of the nervous tissue, and dysregulation of microRNA. Environmental factors like long-term contact with some metal toxicity and pesticides are other common feature of these diseases [8-11]. The main challenge for the treatment 
of such multifactorial diseases is to identify the appropriate and effective targets for obstruction and prevent disease progression [7].

At present, there is no definitive cure for any of these diseases, and most of them are symptomatic treatments performed by western medicines to relieve pain, control symptoms, and improve mobility [5]. In the 1970s, the first targetbased pharmacotherapy groups were introduced to replacement of neurotransmitters, releasing agents, enzyme inhibitors, direct receptor agonists, and second messenger modulators. Among them, dopaminergic drugs to dopamine replacement [12], acetylcholinesterase inhibitors to inhibit the cholinesterase enzyme [13], analgesic drugs to relieve pain [14], and even the surgical treatment such as deep brain stimulation for the management of many movement disorders [15] are the first generation of these drugs that have been used for years, and even many of them have been approved for clinical use. For instances, L-DOPA (L-3,4-dihydroxyphenylalanine) is the first FDA-approved drug for the clinical treatment of PD disease $[16,17]$ and cholinesterase inhibitors are an important class of FDA-approved drugs that have used to treat all stages of AD since 1978 [18, 19]. The second generation of these medicines is designed with the aim of slowing and stopping the progression of this disease, for instance, CERE-120 (adeno-associated virus serotype 2-neurturin) which is in phase I trial for patients with idiopathic PD [20], nonsteroidal anti-inflammatory drugs (NSAIDs) which reduce the risk of AD with long-term use [21], and riluzole for ALS, cerebellar ataxia, and Huntington's disease [22-24].

According to convincing evidence, many of neurodegenerative diseases are multifactorial disorders that involve numerous mechanisms in their etiologies [25]. Thus, conventional therapy strategy with the slogan "a gene, a drug, a disease" more likely not able to suggest an effective pathway toward the development of effective disease-modifying therapies for these diseases. In contrast, concurrent targeting of multiple disease-associated gene and/or protein by engineered compounds that may have several mechanisms of action is needed to targeting the complementary pathways involved in these patients [26]. However, polypharmacy and combination therapy with a composition of several drugs to stop or block the various etiological targets of a disease due to high side effects, multiple toxicities, and possible drug-drug reaction are not proper for some of the neurodegenerative diseases such as $\mathrm{AD}$ that is more common among older adults [26, 27].

An alternative solution is the use of multi-target-directed ligands (MTDLs) or polypharmacology as a drugs that can simultaneously target more than one specific ligand of a disease [28]. For example, memoquin which is a MTDLs based on a polyamine-quinone skeleton discovered by Bolognesi and Melchiorre simultaneously is able to target several mechanisms involved in $\mathrm{AD}$ including aggregation of $\beta$-amyloid peptide $(A \beta)$, producing of oxygen-containing radicals and inhibit activities of acetylcholinesterase (AChE) [29]. In preclinical investigations, decreased $A \beta$ peptide, ROS formation, and even improvement in behavioral deficits were demonstrated after memoquin injection [29]. The complex multifaceted nature of the etiopathology of these diseases precludes the identification of evolutionary relationships between the targets selected for the design of a multi-ligand compound [30]. Also, as neurological disorder progresses, the dose of medication is increased to compensate reduced effectiveness of pharmacological treatments which leads to an increase in side effects [31].

In the search to find treatments for neurological disorders, increase access to enabling technologies in neuroscience and identifying the accumulation of incorrect proteins as one of the characteristics of many neurological disorders and understanding their role in the pathogenesis of these diseases have shifted the focus on what could be done to prevent it. About 20 years ago, gene therapy approaches were proposed as an effective treatment strategy to prevent or induce the expression of specific proteins leading to emergence of new hopes to neuroprotection, neurorestoration, and even correction of the underlying pathogenic mechanism [32]. There are many parameters including disease pathogenesis, spatiotemporal specificity of gene expression, and choice of appropriate vector need to have considered to achieved effective gene therapy for treatment of these diseases [33].

Various strategies have been used to alter the expression of these genes, including replacement of defective genes and changing DNA sequences by gene editing techniques such as zinc finger proteins (ZFPs) and meganucleases and transcription activator-like effector nucleases (TALENs) and the most recent of these techniques, CRISPR/Cas9, with the ability to more accurately and efficiently double-strand breaks (DSBs) at the target DNA [34-36]. Also, alteration of the expression defective genes by post-transcriptional modifications such as RNA interference (RNAi), anti-sense oligonucleotides (ASOs), and catalytic nucleic acids provides new hope in the treatment of neurological diseases [37]. The first in vivo studies to treat neurodegenerative disease by RNAi were performed on HD in 2005 [38]. Harper reported a significant reduction in HTT expression in both mRNA and HTT inclusions, as well as improved behavioral and neuropathological abnormalities after striatum injections of AAVencased anti-HTT shRNA into NLS-N171-82Q transgenic mouse model of HD [38].

As abovementioned, the most common mechanism among various neurological disease is progressive degeneration and death of neuron cells which are never naturally replaced due to the inherent limitation of endogenous regeneration of the central nervous system [5]. Also, none of the current drug options can prevent and slow the progression of the irreversible destruction of neurons [33]. Now, the only proper strategy is cell therapy especially stem cell-based therapy [39]. The results 
from many different preclinical and clinical trials studies from 1980 to the present reveal that stem cell therapy is the only rationale and feasible tool for the regeneration of neural tissue. The main barrier for using the therapeutic potential of these cells is the blood-brain barrier (BBB) [39]. The BBB is a strict, selective semipermeable barrier that maintains the CNS homeostasis by preventing the penetration of nonselective solutes into the brain [40].

Due to the impermeability of molecules larger than $400 \mathrm{Da}$ of $\mathrm{BBB}$, the use of cell-based treatment strategies requires bypassing the BBB via physical or chemical methods, which not only are invasive procedures but may also cause side effects [41]. Classically, there are three ways to improve the delivery of therapeutic compounds to the brain by passing via the $\mathrm{BBB}$, including an invasive method that directly injects the drug by breaching the $\mathrm{BBB}$, and the pharmacological method that is based on several parameters which efficiently affects the ability to passively cross the BBB, such as shape and size $(<400 \mathrm{Da})$, charge (zeta potential), and the type of ligands (lipophilic ligand is better than transport). And the most effective delivery method, the physiological approach, in which the active drug is delivered specifically to the brain through transporters or internalizing receptors that are highly expressed at the BBB surface, such as transferrin and insulin receptors $[42,43]$.

Unlike stem cells, the paracrine factors secreted by them, including growth factors and extracellular vesicles, cross the BBB well due to their small size. As will be explained in detail below, these secreted paracrine factors show similar and even same effects to their source cell [44]. Therefore, they may be introduced as an alternative strategy for the treatment of neurological disorders. In this review, we discuss the therapeutic potential of extracellular vesicles specially exosomes as one of the paracrine factors secreted from stem cells to the treatment of these disease, and we also challenge the strengths and weaknesses of this strategy. In the following, we will review studies based on extracellular vesicle (EVs) and exosomes for the treatment of various neurological disorders. The main focus of this review evaluated the internal potential of stem cellderived EV to the treatment of these diseases, so studies that have been used of modified EV have noted lowly.

\section{Stem Cells}

The discovery of stem cells in the second half of the nineteenth century and their identification as a natural body tool to develop and repair itself revolutionized medical practice and significantly increased the tendency to study them for the treatment of various diseases [39, 45-47]. Since then, the list of clinical applications of stem cells in the treatment of various diseases has been steadily increased [48]. Stem cells are unique undifferentiated cells that demonstrate special properties including high proliferation, differentiation, and self-renewal potentials, compared to specialized cells [49, 50]. Stem cells are found in embryonic and adult tissues including adipose, the bone marrow, blood, CNS, and dental tissue $[50,51]$.

Based on the cellular lineages that stem cells produce and the source in which stem cells are derived, stem cells divided into embryonic stem cells (ESCs), mesenchymal stem cells (MSCs), progenitor cells, and induced pluripotent stem (iPS) cells [52]. Also, stem cells are categorized by their differentiation potential into different cell types including totipotent, pluripotent, multipotent, or unipotent [53]. The zygote cells are known as the only totipotent stem cells that can differentiate into any type of cell in the embryo as well as extraembryonic cells (placenta) [54]. ESC cell are pluripotent stem cells that are able to give rise to differentiated cell types derived from all three germ layers of the embryo derived from the inner cell mass of a blastocyst [55]. Remarkable ability of unlimited self-renewal and differentiating into neurons introduced ESCs as an interesting avenue for research in neurodegenerative disorders [56]. But because their production is associated with the destruction and damage of the fetus, their clinical use faces a major ethical dilemma [57]. IPSCs are artificial pluripotent stem cell derived from the reprogramming adult non-pluripotent somatic cells [58, 59]. Multipotent stem cells are more limited than pluripotent cells that can only differentiate into a few types of specialized cells present in a specific tissue. These cells can be isolated from various sources of adult stem cells and cord blood stem cells [49]. Mesenchymal stem cells (MSCs) are heterogeneous multipotent stem cells which able to differentiate into lineage of mesenchymal tissues including bone, cartilage, adipose, muscle, and tendon. Multipotent stem cells specially MSCs are the main cellular source for autologous therapies without worry, including immune responses and ethical issues such as those associated with ESC [60]. Various evidence suggested that adult stem cells are present in the peripheral circulation at a very low-frequency steady-state for repair and maintain tissue homeostasis [61]. In general, MSCs provide the profitability of tissue-specific stem cell niche to their function and promote tissue regeneration [61]. Several weaknesses such as limited proliferative capacity, difficult to standardize, loss of differentiation potential, and therapeutic efficacy during multiple expansion hinder therapeutic application of MSCs. Previous studies have produced single-cell MSCs derived from human induced pluripotent stem cells (iPSCs) with MSC-comparable ability including immunomodulatory effects, self-renewal and repair of injured tissue, and paracrine ability [62]. Pericytes or perivascular cells are heterogeneous and multifunctional cells present in every vascularized tissue around the body like endothelial cells and have critical roles in the stability and angiogenesis of blood vessels [63]. Pericytes are phenotypically similar to stem cells in terms of cell surface 
markers (CD146+/CD34-/CD45-/CD56-) and functional properties including their ability to self-renew and even differentiate into a variety of mesenchymal cell types such as chondrocytes, adipocytes, osteocytes, and myocytes in culture which reinforces the hypothesis that the two cell types may be the same or at least very closely to each other [64, 65]. Also, several studies such as that of Kunisaki et al. demonstrated that pericytes are critical source of niche factors essential for hematopoietic stem cells maintenance in the bone marrow [63, 66]. Such ability suggests pericytes being a suitable replacement candidate for MSCs without its restriction. For example, Yuan's team revealed therapeutic potential of exosomes derived from pericytes to protect the blood-spinal cord barrier under hypoxia condition. The evaluation underlying the mechanism demonstrated that taken up pericyte-derived exosomes by endothelial cells ameliorated its function through overexpression of junction protein, attenuating the apoptotic response and extent behavioral recovery after spinal cord injury mice model [67]. The last group of stem cells is unipotent stem cell with self-renewal ability that differentiate into only one specific cell type forming a single lineage [68].

The landmark stem cell clinical trials were way back in the 1980s when fetal-derived neural tissue transplants were used in the treatment of PD in Mexico [69]. Although variable results have been obtained from this study, it has provided a promising strategy based on replacement or protective therapy for the treatment of various neurological diseases including $\mathrm{PD}, \mathrm{HD}, \mathrm{AD}$, and ALS [70-73]. In addition to the known stem cell properties that are mentioned above, the distinctive therapeutic features of them including potential for differentiation, ability of homing and migration to the site of injury, secretion of neurological factors such as basic fibroblast growth factor (bFGF), brain-derived neurotrophic factor (BDNF), and vascular endothelial growth factor (VEGF) and other factors with anti-inflammatory and anti-fibrotic effects either freely or packaged inside extracellular vesicles make them a powerful tool for tissue healing and treating neurological diseases [74-76].

\section{Exosomes}

Membrane-bound extracellular vesicles (EVs) originating from the endocytic system are called exosomes [77]. According to the International Society for Extracellular Vesicles (ISEV), based on origin, size, and cargo, EVs are divided into three categories including exosomes, microvesicles, and apoptotic bodies [78]. Among them, exosomes are the smallest group with the nanoscale size of 40-150 nm, which are often employed by two methods: nanoparticle trace analysis (NTA) and dynamic light scattering (DLS) $[79,80]$. In addition to another hallmark property of exosome that distinguishes from other EVs is their biogenesis.
Exosomes, unlike microvesicles which bud directly from plasma membrane and apoptotic bodies that formed during apoptosis, have endosomal origin [81]. Exosomes are the product of the fusion of multivesicular bodies (MVB) with the plasma membrane (Fig. 1) [82]. Multivesicular bodies (MVB) are intracellular multivesicular compartment that are produced by invagination of the endosomal membrane [83]. Nevertheless, since there are no definitive markers for the exosome and none of the isolation techniques can definitively purify the exosome from microvesicles, in more time, both are defined as extracellular vesicles (EVs) [84, 85].

For the first time, Pan and Johnstone (1983) discovered exosome as relatively uniform-sized particles during sheep reticulocyte maturation [86]. It was later found that not only the most cells such as neurons, epithelial, endothelial, and stem cell [87] secrete exosomes but also some cells including immune cells and mesenchymal stem cells (MSCs) produce more exosomes than other cells [88]. Also, exosome is present in various body fluids including blood, urine, saliva, amniotic fluid, breast milk, synovial fluid, broncho-alveolar lavage fluid, and cerebrospinal fluid [89]. Structurally, exosomes are composed of a phospholipid bilayer and cytosolic contents which are representative of its donor cells [90]. Exosome lipid bilayer membrane besides ensuring membrane stability and durability, involved in other processes including its production during budding and its uptake by the target cell [91]. For instance, phosphatidylserine is involved in budding and fusion of the exosomes through increased flexibility due to its conical shape [92]. In addition, lipid messengers and cellular signals such as phosphatidic acid, diglycerides, and ceramides of exosomal membrane are involved in both pathways dependent and independent of ESCRT machinery in exosome biogenesis and also in packaging of biological cargo into exosomes [93, 94]. Other concentrated compounds in exosomal membranes include tetraspanins (CD9, CD63, CD81, CD82), lysosomal-associated membrane protein (LAMP) 1, 2, major histocompatibility complex (MHC) I, II, intercellular adhesion molecule 1 (ICAM-1), CD13, integrin, annexin, lipid rafts, fusion proteins like flotillin, TSG101 (Tumor Susceptibility Gene 101), heat-shock proteins HSC70 and HSC90, and GTPases [95]. These surface proteins with cytosolic loads are not only considered as an indicator of the separation of exosomes from other extracellular vesicles but also act as a source of biological markers for various pathological conditions [80, 95]. Cytosolic biomolecule component of exosome is dependent on the cell type origin, including protein, lipid, cytoskeleton protein, signaling molecules, enzymes, and genetic molecules. Analysis of exosome nucleotide content by next-generation sequencing (NGS)-based studies showed that in addition to mRNA and some short DNA, exosomes contain various non-coding RNA including miRNA, ribosomal RNA (rRNA), and transfer RNA (tRNA) [96]. Also probably this is due to production 
Fig. 1 Biogenesis of exosomes and its contents
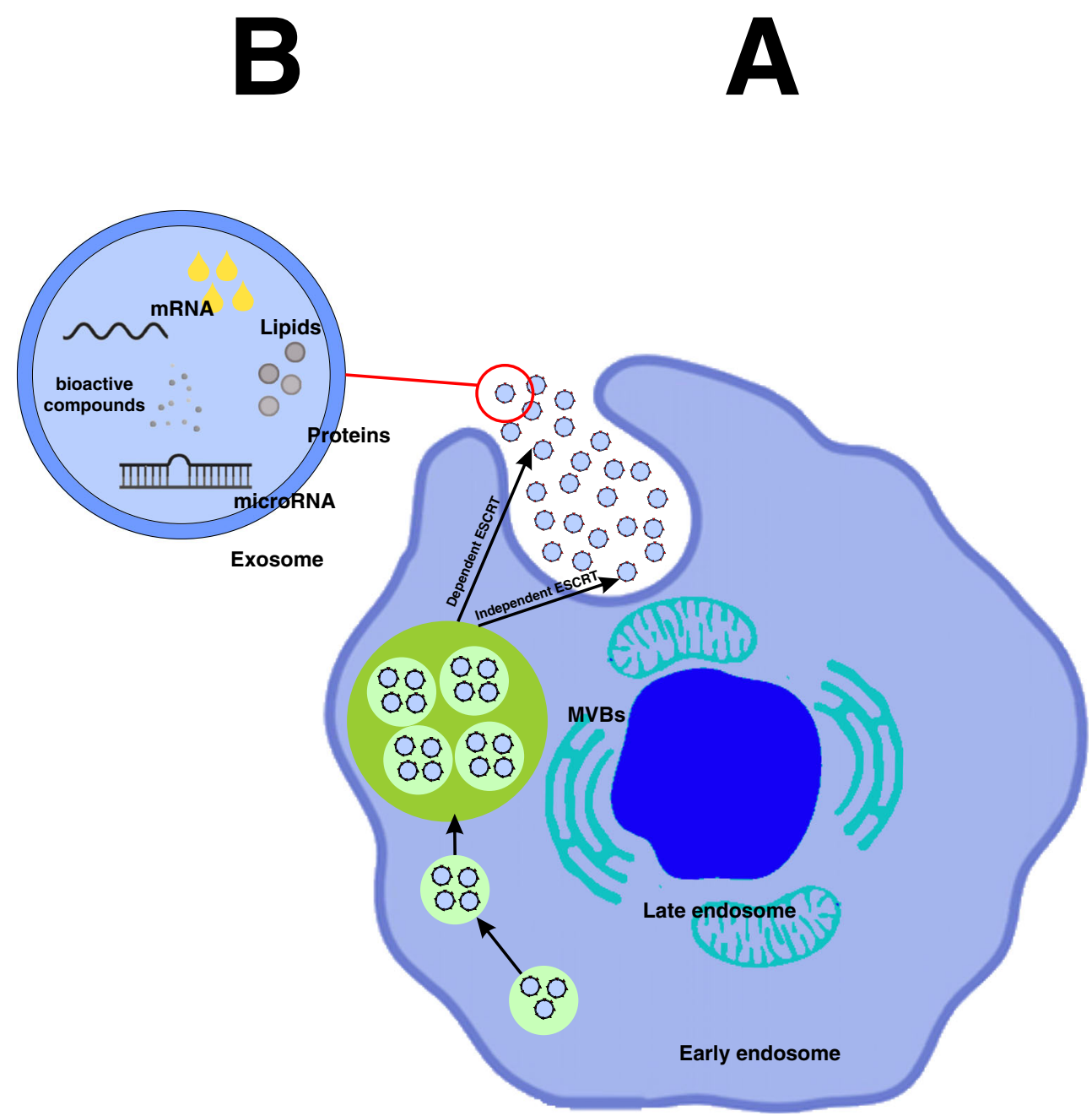

of exosome through invagination of the endosomal membrane, loading exosomes with plasma membrane and cytoplasmic proteins more than nuclear and mitochondrial proteins [97]. Today's various techniques such as flow cytometry, mass spectrometry (MS), western blotting, and immuneelectron microscopy were used for characterization of lipid and protein contents of exosome [90].

Proteomic studies have so far identified more than 4000 different types of proteins in exosomes [98]. The results of such studies show that the protein contents of exosomes derived from different sources are similar which suggested protein loading is controlled by defined mechanisms [90]. Although exosomes are homogeneous and many of their contents are shared due to their endocytosis origin, some of the components may differ depending on their source and the physiological conditions of the donor cell [99]. However, the exosome composition is not completely in concordance with the donor cells and inverse donor cell; some components are rich or poor. This indicates that the exosome loads are selected and performed by different pathways [85, 92]. Analyzing the cargo and content of the exosome is important because, as we know, the exosomes are selectively taken up by other cells (neighbor and distal cells) after the transfer to the extracellular matrix [100]. Although there are still many ambiguous points about the biological function of exosomes, the cell-to-cell communication is considered to be their most important function [101]. In other words, exosomes act as the most important communicator between cells that have no direct contact with each other [102]. Exosome membrane proteins as ligands can bind directly to target cell membrane receptors [103]. The most common visualization techniques of the cellular uptake of exosomes include lipid-specific fluorescence dyes such as PKH67, PKH26, DiI, and DiD and/or synthetic compounds like CFSE and CFDA that acquire fluorescence only after changes in cytoplasmic conditions [85, 104]. Horizontal translocation of exosome biomolecule cargo specially non-coding miRNA and mRNA results in epigenetic reprogramming of the target cell [105]. However, the pivotal role of exosomes in the progression of neurodegenerative disorders through the transport of specific toxic proteins such as $\alpha$-synuclein, $\mathrm{A} \beta$, and $\mathrm{mHTT}$ from producer cell to other cell has been identified [106-109]. 
Exosomes are key tools for transmitting paracrine signaling to other cells [110]. Numerous studies have confirmed the two-way transmission of blood circulation between the brain and the bloodstream [43]. Use of blood-isolated neuron-derived $\mathrm{EV}$ as biomarker for various neurodegenerative diseases is a clear witness to cross EV from the brain to the bloodstream [111-113]. Although, cross from BBB is a critical advantage of exosome that significantly increases the interest in using EXOs as delivery therapy tools, the mechanical details of the process of BBB passage through exosome are not yet known. According to the result of high-tech methods, exosomes can interact with their target cells of the BBB by five possible theoretical routes including interaction with $G$ protein-coupled receptor (GPCR) on the cell surface, releasing content of exosome in the cytoplasm receiving cell by fusion with the cell membrane, receptor-mediated transcytosis, including receptor lipid raft-mediated and micropinocytosis $[114,115]$. Zhao and his colleague elucidated that exosome derived from cell line in the inflammatory stroke-like conditions, but not on normal condition, can cross brain microvascular endothelial cells (BMECs) through endocytosis and colocalize with endosomes including receptor-mediated transcytosis, lipid raft-mediated and micropinocytosis [116]. In addition, as previously mentioned, EVs have a wide variety of different molecules on their surface, some of which depend on its cell type origin. Among them, integrins, tetraspanins, and some ligands were discovered as potential mediators for interaction of exosome with receiving cell [114]. For instance, Terasaki's team revealed that integrin $\alpha 5$, integrin $\alpha \mathrm{V}$, and CD46 on the surface of the human blood-brain barrier endothelial cells (hCMEC/D3 cells) are responsible for internalization of exosomes [117]. Such studies elucidated an inevitable interaction between $\mathrm{EV}$ and different members of a BBB within the brain microvasculature that mediated by surface markers in both normal and pathological conditions [114], because some studies like Dozio et al. found that cells pretreated with pro-inflammatory cytokines such as TNF $\alpha$ increase proteins associated with TNF $\alpha$ and NF- $\mathrm{KB}$ signaling pathways in cells and their derived EV [118]. Also, inflammatory environment in neurodegenerative disorders through BBB failure increases its permeability [118]. Finally, the heterogeneous nature of exosomes leads to the presence of various proteins on their surfaces that, after interaction with the cell surface marker, facilitate their entry into receiving cells. Therefore, revealing the mechanism by which exosomes cross the blood-brain barrier requires more information about the physiology and dynamics of EV and BBB. After endocytosis targets the cell, exosomes have two different fate in the target cell, and they are transported to adjacent cells via transcytosis or destroying as a result of integration with lysosomes [103]. In vivo behaviors such as stability and biodistribution of exosomes are other challenging issues in their diagnostic and therapeutic applications. In many studies, the higher stability of biomolecules (miRNAs and protein) in the exosome due to their protection against extracellular degradation has been considered as a useful biomarker [119]. In order to better understand the in vivo behavior of exosomes, the valuation of pharmacokinetic properties of exosome is essential. The pharmacokinetic properties of exosomes are elucidated based on the biodistribution or tissue distribution and cellular uptake [120]. Primary result revealed that biodistribution rate of exosomes is affected by the route of administration and displays a dose-dependent manner [88].

Some of intrinsic properties of unmodified exosome improve its stability in peripheral circulation including (i) small size: exosomes are nanoscale vesicles $(40-150 \mathrm{~nm})$ that are well able to escape from engulfed by phagocytic cells [121], (ii) surface marker: the most possible mechanism degradation of membrane vesicle-like apoptotic bodies and microvesicles in the periphery is opsonization or lysis by the complement membrane attack complex. Many studies found that EVs are an exception in this respect and have a capacity to survive in the periphery circulation. Exosomes due to distinct production route express the high level of the GPI-anchored complement regulators such as CD55 and CD59 that able to suppress complement-mediated lysis and promote survival in the extracellular environment [122]. (iii) Biocompatibility: the biological origin of exosomes significantly decreases the immune response and has more biocompatibility unlike nanodrug delivery systems such as liposomes [123, 124].

Evaluation of the tissue distribution of exogenously administered exosomes by different labeling methods elucidated that homing tissues of exosomes derived from cell culture after intravenous injection in the mice model are those of the liver, spleen, gastrointestinal tract, and lungs which can differ based on cell-of-origin [125], while intravenous injection of exosomes isolated from body fluid samples mostly accumulated in the liver. Also, other studies suggested that tissueresident macrophages in the spleen and liver probably have important role in the taking up and clearance exosomes after systematic injection [120,126].

Undoubtedly, this is performed via the identification of surface molecules on the exosome by macrophages and other cells [127]. For instance, the presence of negatively charged molecule like phosphatidylserine (PS) on the surface exosome like apoptotic bodies leads to identification and take-up by macrophages [128]. In addition, sialic acid and integrin $\alpha 4 \beta 6$ are recognized as key molecules that are involved in the take-up of exosome by macrophages in the spleen and lung, respectively $[129,130]$.

As abovementioned, the therapeutic potential of exosomes depends entirely on their biological cargoes which are dependent on the cell type origin [131]. Having such therapeutic burdens may be intrinsic, such as exosomes derived from cells with therapeutic activity like stem cells have anti-inflammatory and neuroprotective properties [6, 132-154]. Discovering such abilities of exosomes in addition to weak stimulation of the immune system, crossing of the BBB, and long-term stability in the 
circulation system enhanced their potential in creating new treatment strategies for a variety of diseases specially in the central nervous system (CNS) regenerative medicine field [89].

\section{Therapeutic Potential of Stem Cell-Derived Exosome in Stroke}

Stroke is a major neurological disorder with a high rate of incapacitation and fatality worldwide that prevents the proper functioning of parts of the brain [155]. Major strokes are ischemic strokes [156]. An ischemic stroke happens when blood flow becomes blocked via the artery that provides oxygen-rich blood to the brain. Blood clots often cause the blockages that results in ischemic strokes [157]. Intravenous tissue plasminogen activator (IV-tPA) and endovascular thrombectomy (ET) are the current gold standards for the treatment of acute ischemic stroke [158, 159], because both treatments are FDA approved for curing stroke [158, 159]. Due to the limited access of many patients to these drugs, many patients are still untreated and their possible treatment is done through mechanisms such as neurorehabilitation and endogenous neuroregeneration mechanisms [160, 161].

One of the new research strategies being pursued by researchers with the ultimate goal of treating this disease is the use of extracellular stem cell-derived vesicles. Thus, there is a lot of in vitro and preclinical studies which were performed using EVs derived from stem cells in this field. In 2013, the MSC-derived exosomes were first administered using intravenous injection followed by neuronal remodeling and functional recovery in a rat stroke model. It has been indicated by Xin et al. that exosomes may lead to improved synaptic plasticity, angiogenesis, neurite remodeling, and neurogenesis on ischemic boundary zones in a rat model of stroke [162]. Webb et al. demonstrated the recovery capacity of human neural stem cell-derived EVs in a pig ischemic stroke model. They reported the neuroprotective properties of NSC-EVs which lead to improve behavior and mobility in stroked pigs by removing intracranial hemorrhage and reducing the volume of the cerebral lesions and brain swelling compared with control pigs [163]. Another study by the same researchers showed the ability of NSC-EVs to ameliorate cellular, tissue, and functional recovery in the middle-aged thromboembolic stroke model that are related to changing the immune response [132]. Intravenous administration of MSC-EVs enhanced white matter integrity via inducing axonal sprouting, remyelination, and oligodendrogenesis after subcortical stroke in rats [154].

Due to the possibility of effective transfer of microRNAs involved in cellular and molecular processes such as cellular senescence, telomere length, and circadian rhythm into stem cells, EVs harvested from these cells, which targeted microRNAs that may be overexpressed in them, could lead to improvements in age-associated diseases including stroke and AD [164]. Thus, in several studies, the different group used exosomes to modify the increased expression of microRNAs, such as miR-17-92 cluster, miR-184, miR-210, and miR-133b, microRNAs which have neuroprotective properties [138, 140, 142, 165]. The results by Xin et al. demonstrated influence of intra-arterial injection of exosome harvested from microRNA 133b-overexpressing MSCs into a middle cerebral artery occlusion area in a cerebral artery rat model. Consequently, neuronal plasticity and amelioration of functional recovery occurred as a result of a decreasing gene levels of phosphatase and tensin homolog (PTEN) in addition to activating downstream phosphatidylinositol-3-kinase/protein kinase $\mathrm{B} / \mathrm{mammalian}$ target of rapamycin (PI3K/Akt/mTOR) signaling following inactivation of glycogen synthase kinase (GSK)-3 $\beta$ in a rat model of stroke [140]. Apart from capabilities of stem cell-derived EVs in neuroprotection and nerve regeneration that were discussed earlier, these biomolecules have shown inhibitory effects on proliferation of immune cells such as B, T, and natural killer (NK) cells [139]. Doeppner et al. reported the induced post-ischemic neurological recovery via stimulation of angioneurogenesis mediated by bone marrow MSC exosome in stroke mice modal [139].

Besides, Barzegar et al. (2020) reported that exosomes harvested from angiotensin-converting enzyme 2-expressing human placental MSCs led to increased neuroregenerative poststroke effects in an acute ischemic stroke model. In addition to neurological recovery, they also demonstrated that these exosomes could provide support against detrimental effects induced by ischemic stroke [166]. Similarly, an animal preclinical experiment concluded that small EVs obtained from bone marrow MSCs stimulated neurological recovery by reducing brain infiltration of leukocytes with a capacity of ischemic neuroprotection and neurological deficit reduction [167]. Moon and colleagues investigated the therapeutic potential of MSC-EVs on rat stroke model. They find that endogenous cargo of exosomes such as miRNA-184 and miRNA-210 are responsible for the induction of neurogenesis and angiogenesis by MSC-EVs [142].

Deng et al. documented that increased miR-126 expression in MSC exosomes can significantly enhance TNF $\alpha$, IL- $1 \beta$, and IL-6 expression, while decreasing neuronal apoptosis in a middle cerebral artery occlusion mouse model [168]. A research experiment on mouse models with focal cerebral ischemia demonstrated that intravenous administration the exosomes derived from primary mouse neural stem cells (NSCs) or human induced pluripotent stem cell-derived cardiomyocyte (iCM) is a therapeutically effective agent for the amelioration of symptoms and lesions of experimental stroke disease, including reduced infarct volume, due to its capability in maintaining astrocyte function, as well as the neuroprotective properties [141]. In one in vivo study, it was observed that MSC exosomes in conjunction with rosuvastatin can be 
applied to the reduce infarct volume and decrease the NLRP1 and NLRP3 gene expression as well as increase functional relief and neuroprotection on rat model of ischemic stroke with middle cerebral artery occlusion (MCAO) [169]. Furthermore, it was indicated that small EVs obtained from human iPSC-derived MSCs may regulate the angiogenesis, through inhibiting in STAT3-dependent autophagy after ischemic stroke in a mouse model [170].

One of the important issues that should be considered in order to use EVs in the treatment of stroke is the optimal time to use EVs after the onset of the disease. On the other hand, studies show that the use of EVs in the short time after the onset of stroke shows better results $[57,162]$. Xin et al. have shown that administration of MSC-derived exosomes could improve functional recovery as well as ameliorate neurite remodeling in the first $24 \mathrm{~h}$ after stroke onset [162], while injection of MSC-derived exosomes 3 days after stroke onset reduces the hemorrhage and $\mathrm{BBB}$ leakage, in addition to an enhanced white matter remodeling [171]. Sun et al. during a study evaluated the anti-ischemic properties of two types of $\mathrm{SC}$-derived exosome including NSC and iCM in the in vitro and in vivo model of ischemic stroke. Primary mouse astrocyte or neuronal cultures were subjected to in vitro ischemic injury by oxygen-glucose depletion (OGD). NSC-derived exosomes induced significant protection in OGD-exposed astrocytes [141]. Taken together, such results clearly reveal the successful function of cell-free therapeutic strategy-based stem cells in the treatment of stroke-related impairment.

\section{Therapeutic Potential of Stem Cell-Derived Exosome in AD}

$\mathrm{AD}$ is the most well-known cause of dementia that mainly affects the elderly population [172]. This neurodegenerative disorder is a progressive and irreversible brain disorder that affects cognitive function (thinking, reasoning, and remembering), personality, the thought content, and behavior [173]. Three hypotheses of $\mathrm{AD}$ including extracellular accumulation of beta amyloid peptide $(\mathrm{A} \beta)$ called amyloid plaques, formation of neurofibrillary tangles (NFT) due to intracellular deposition of hyperphosphorylated tau protein, and chronic neuroinflammation are the most acceptable scientific hypotheses of pathological features in AD [174]. Such abnormal accumulations of protein inside and outside nerve cells cause neurotic communication disorders and destruction of specific neurons [175].

There is a general agreement that the accumulation of $A \beta$ peptide, the main composition of amyloid plaque, in neurons is responsible for the early stages of pathogenesis in $\mathrm{AD}$ [176]. Numerous studies show that the main site of $A \beta$ production in neurons is multivariate bodies (MVBs) [177]. According to numerous studies, the internal vesicles (ILVs) that make up
MVBs have two destinies: degradation due to delivery to the lysosomal lumen or secretion into surrounding extracellular matrix as exosomes through fusion with the plasma membrane [178]. These results emphasize the role of exosomes in the development of $\mathrm{AD}$ through the spread of $\mathrm{A} \beta$ deposits [179]. The use of exosomes as a biomarker for early detection of AD or as a tool for delivery of therapeutic molecules such as small drugs, siRNA, and miRNA has been investigated in several studies [180, 181]. For example, Saman et al. used cerebrospinal fluid (CSF)-derived tau-containing exosomes for early detection of AD [182]. Also, of the CSF-derived exosome carries $A \beta$ and $p$-tau [183, 184], the identification of both potential biomarkers in CSF-derived exosomes may lead to increased value of used marker for early detection of AD [184]. Clark et al. confirmed $86 \%$ increased sensitivity and specificity when using combination markers (CSF p-tau and $A \beta$ ) to diagnose $A D$ [185]. In addition, miRNA expression profile analysis of exosomes can provide relatively accurate insights into the pathogenesis of AD patients. Many studies exploited of exosomal miRNAs from various body fluids including plasma and CSF as biomarker for $\mathrm{AD}$ diagnosis $[186,187]$. One study found that CSF-derived exosomes had higher levels of two microRNAs, including miR-9-5p and miR-598, in AD patients than healthy controls [188]. However, CSF has long been used to separate markers, and the relative aggressiveness of the CSF collection has limited its use; thus, plasma may be an easier and more accessible alternative [189]. Also, the biological content of plasmaderived exosomes has shown high accuracy for early detection of AD [111]. For instance, comparison of plasma exosomal protein between $\mathrm{AD}$ patients and control group showed high levels of neuron-derived proteins including $A \beta$ and total tau in patients with $\mathrm{AD}$ [184]. There are many such studies, but our main focus is on therapeutic applications.

Neuroprotection activity of neuron-derived exosome have been confirmed in several studies. For example, glia-derived exosomes promote neuroprotection against oxidative stress by transduction of synapsin I, an oligomannose-carrying glycoprotein [190]. The results of investigating the mechanisms associated with exosomes in $A \beta$ clearance indicate that the neuron-derived exosomes after injection into the brain of $\mathrm{AD}$ transgenic mouse models helped in $A \beta$ peptide removal through two major mechanisms. Exosomal surface prion receptor can attach to amyloid plaques and convert them into nontoxic forms due to conformational changes. In addition, the exosome accelerates the uptake of extracellular plaque of A $\beta$ by microglia [191, 192]. Unfortunately, many of the studies were performed on therapeutic application of exosome secreted by MSC.

Exosomes secreted by MSC derived from connective tissue including adipose tissue and bone marrow not only cross the blood-brain barrier very well [116] but also, due to the presence of neprilysin enzymes especially in the MSC-derived 
EVs from adipose tissue, effectively cleave extracellular and intracellular A $\beta$ peptides in the brain [193]. Similarly, intracerebral injection of bone marrow MSC-EVs into the neocortex of APPswe/PS1dE9 mouse model of AD leads to decreased $A \beta$ levels and plaque load and number of dystrophic neurites in both the hippocampus and cortex by two direct and indirect mechanisms. In the direct mechanism, MSC-EV interact with $\mathrm{A} \beta$ plaques through its lipid membranes and increase plaque phagocytosis by microglial cells. Also, MSCEVs carry the neprilysin, a proteolysis of $A \beta$ plaques, indirectly reducing intracellular $A \beta$ deposits [146]. Another study showed that injection of MSCs or MSC-derived EV increases the resistance of hippocampal neurons to the deleterious effects of oxidative stress and $A \beta$-induced synapse damage [194]. De Godoy and his colleagues proposed several possible mechanisms to explain the neuroprotection effects of MSCderived $\mathrm{EV}$ and $\mathrm{MSC}$ transplantation against $\mathrm{A} \beta$-induced neuronal damage, including decreased extracellular $A \beta$ oligomer level due to high endocytic capacity of MSCs, secretion of EVs containing the antioxidant enzyme such as catalase, and paracrine action via extracellular release of antiinflammatory cytokines and trophic factors including IL-6, IL-10, and VEGF [194]. Similarly, to investigate the immune regulatory properties, EVs secreted by cytokinepreconditioned MSC with (TNF $\alpha$ and INF $\gamma$ ) were injected via intranasal in a $3 \times \mathrm{TTg} \mathrm{AD}$ model. Overexpression of COX2 and IDO, reducing the secretion of IL- 6 and IL- $1 \beta$, and conversely increasing the secretion of IL-10 which induces the M2 phenotype have been reported as results of this study [147]. In an interesting study carried out by Perets et al., the critical role of inflammation in the attraction and migration of exosome derived from MSC toward different brain pathologies, including stroke, autism, $\mathrm{PD}$, and $\mathrm{AD}$ through in vivo neuroimaging of exosome-based method has been shown. They also demonstrated remaining and increased accumulation of MSC exosome in certain areas after $96 \mathrm{~h}$. This is a confirmation for homing abilities of MSC exosome in various pathologies[135].

Similarly, in 2019, Bodart-Santos et al. investigated the neuroprotective action of human Wharton's jelly mesenchymal stem cell-derived EV (hMSC-EVs) on the in vitro culture of hippocampal cells against oxidative stress and synapse damage induced by $\mathrm{A} \beta$ oligomers. They reported three results from their observation: (1) the total number of EVs taken up by the hippocampal cells increases significantly in the presence of $A \beta$, (2) HMSC-EVs increases the resistance of hippocampal neurons to damage caused by $\mathrm{A} \beta$, and (3) neuroprotection effect of hMSC-EVs is dependent on enzymatically active catalase. They also demonstrated the ability of EV to inhibit $A \beta$-induced damage by regulating the function of astrocytes (as a key player in inflammatory responses of the CNS) and decreasing ROS production. Based on these results, they introduced hMSC-EV as a candidate for the treatment of AD [195].

Another study performed by Cui and his group in 2018 investigated recovery from cognitive impairment in APP/PS1 mouse model of $\mathrm{AD}$ by exosomes derived from hypoxiapreconditioned mesenchymal stromal cells. Although both MSCs and hypoxia-preconditioned MSC-derived exosome reduced intracellular and extracellular deposition of $\mathrm{A} \beta$ oligomers, but hypoxia-preconditioned MSC-derived exosomes obviously ameliorates learning and memory deficits through reduced serum levels of pro-inflammatory cytokines (IL-1 $\beta$ and TNF- $\alpha$ ) and vice versa, increased level of inflammatory cytokines (IL-4 and IL-10), and also decreased inflammatory responses via repression of the function of astrocytes and microglia. In addition, the activated levels of STAT3 and NF- $\mathrm{KB}$ in the brain of transgenic mice treated with hypoxia-preconditioned MSC-derived exosomes are reduced [196].

Intracerebral injection of BM-MSC-EVs into the neocortex of APPswe/PS1dE9 mouse model of AD leads to decreased $A \beta$ levels and plaque load and number of dystrophic neurites in both the hippocampus and cortex by two direct and indirect mechanisms. In the direct mechanism, MSC-EVs interact with $\mathrm{A} \beta$ plaques through its lipid membranes and increase plaque phagocytosis by microglial cells. Also MSC-EVs carry the neprilysin, a proteolysis of $\mathrm{A} \beta$ plaques, indirectly reducing intracellular $A \beta$ deposits [146]. Another study revealed the similar effect of MSC exosomes to MSC in inducing neurogenesis and restoring cognitive function lost in mouse model of AD [136].

A recent study conducted by Li and colleagues sheds light on the capacity of neural stem cell-derived EV in ameliorating the cognitive dysfunction in APP/PS1 mice AD. The majorly reported results of this study demonstrated that the mitochondrial function-related factors, SIRT1, as well as synaptic proteins overexpressed while oxidative damage markers, inflammatory cytokines, and the microglial marker significantly decrease compared to the control group[148].

Another study used heat-shock neural stem cell-derived exosome in the treatment of mouse model AD that finally restarts cognitive disability and improves motor function [153].

Although MSC-EV has been used to treat AD in most studies to date, according to two recent studies, different stem cell sources have therapeutic potential to improve AD-induced cognitive dysfunction through various mechanisms such as reducing intracellular and extracellular $\mathrm{A} \beta$ deposition oligomers.

\section{Therapeutic Potential of Stem Cell-Derived Exosome in PD}

PD is a chronic and progressive neurodegenerative disorder which severely affects the dopamine-producing neurons 
[197]. In addition to the loss of dopaminergic neurons, the formation of the Lewy bodies (LB) due to the deposition of $\alpha$-synoklin protein in the cytoplasm of neurons is a prominent feature of PD pathology [198]. Like other brain disorders, there is not yet any effective treatment to delay the progression of PD, and current standard treatments such as levodopa (LDOPA), a direct precursor of dopamine, are only used to reduce symptoms [199]. Also, in order to improve efficiency and overcome side effects, a combination of levodopa with other PD pharmacological treatments is often used [200]. Surgery to deliver electrical pulses to neurons is another PD treatment that is effective in relieving symptoms [201]. In recent years, the effectiveness and efficiency of nonpharmaceutical treatment approaches, including gene therapy, microRNAs, stem cell therapy, and its derived exosomes, have been evaluated for PD treatment [198, 202].

Protein analysis of MSC secretome or condition medium, what MSCs release, explains its inherent and potential therapeutic properties against PD. The survey of the effect of the human MSC (hMSCs) condition medium in the transgenic rat model of PD reveals the potential of HMSC-secretome in increasing dopaminergic neurons, partial return of motor deficits, and histological symptoms [203]. Similarly, the comparison of the neuroprotective effects between transplantation of hBM-MSCs and its derived secretome, in a study in 2019, demonstrated that secretome of hBM-MSCs rather than cell transplantation effectively rescue dopaminergic neurons in the 6-hydroxydopamine (6-OHDA) rat PD model [204]. Also, several studies showed neuroprotective effects of the MSC conditional medium performed through the cleavage of extracellular $\alpha$-synuclein, which is a key element in the progression of PD. For instance, in vitro and in vivo model testing showed that the presence of matrix metalloproteinase 2 (MMP-2) in MSC-secretome by extracellular $\alpha$-synuclein degradation led to a decrease in insoluble forms of $\alpha$-synuclein oligomers and an increase in neural viability in PD disorders [205].

The importance of such studies lies on the fact that EVs especially exosomes are an integral part of secretome of cells, and so undoubtedly, some of such effects are dependent on their secreted EVs. For example, Jarmalavičiūte and his colleagues investigated the paracrine neuroregenerative properties of microvesicles and exosomes secreted from dental pulp stem cells from human exfoliated deciduous teeth (SHEDs) on human dopaminergic neurons. They revealed that exosomes but not microvesicles derived from SHEDs were able to inhibit the apoptosis induced by 6-OHDA in human dopaminergic neurons and finally suggested the use of exosomes derived from SHEDs as therapeutic strategy for treatment of PD [206]. Another studies of the same group used EVs derived from SHEDs to improve motor symptoms on unilateral lesion models of PD induced by 6-OHDA. The obtained result demonstrated that injected EVs not only able to stop the gait impairments induced by 6-OHDA but also improved motor function which is due to normalized striatal tyrosine hydroxylase $(\mathrm{TH})$ activity and expression [152].

In PD, like other neurological diseases, the expression of miRNA profiles is considered as a useful tool for diagnostic and therapeutic purposes [207]. For instance, miR-433 and miR-16-1 have PD-related pathogenic processes that increase the levels of $\alpha$-synuclein [208]. Also, downregulation of miR$34 \mathrm{~b} / \mathrm{c}$ and upregulation of miR-494 and miR-4639-5p have negative and positive effects, respectively, on DJ-1 protein expression (as protector of mitochondrial oxidative damage) [209, 210]. In addition, MSC-derived exosomes induce neural differentiation through the transmission of endogenous and exogenous miRNAs. For example, Lee et al. confirmed the differentiation phenotype in human neuroprotective cells (NPCs) and upregulation of glutamate transporters in both cell NPC and astrocytes, after delivering two exogenous miRNAs including miR-124 and miR-145 by MSC-derived exosomes [211]. In another example, it has been observed that, although, the miR-133b is significantly reduced in PD patients, it is enriched in MSC-derived exosomes, and in vitro and in vivo testing revealed that the transfer of miR-133b by MSCderived exosomes led to the growth of neurons [212]. Shin et al. in 2017 identified miR-17-92 clusters in MSC-derived exosomes with neurogenesis activity that led to stimulation of oligodendrogenesis and improved neuronal function [165]. Despite limited research, the present findings have well demonstrated the beneficial effects of different stem cell sources (MSC and dental SC) in the treatment of PD based on their endogenous EV load.

\section{Therapeutic Potential of Stem Cell-Derived Exosome in Multiple Sclerosis Disease}

Multiple sclerosis (MS) which is an inflammatory demyelination in gray andwhite matter of the central nervous system is the leading cause of non-traumatic neurological disability among young adults especially women [213]. In addition to demyelination and inflammation in the brain and spinal cord, MS characteristic lesion disruptions of the $\mathrm{BBB}$, loss of oligodendrocytes, reactive gliosis, and axonal and neuron degeneration are the other pathological biomarkers of this heterogeneous disease [214]. However, it is generally accepted that activation of peripheral self-reactive Th1 pro-inflammatory cells and attacking the myelin sheath in the CNS by crossing of the BBB is the main mechanism of inflammatory and degenerative properties of MS $[214,215]$. While the pattern of neurological damage in each person with MS is unique, the National MS Society (NMSS) divides the disease into four main types including clinically isolated syndrome (CIS), relapsing-remitting MS (RRMS), primary progressive MS (PPMS), and secondary progressive MS (SPMS). More than $80 \%$ of people with MS are diagnosed with RRMS, which 
eventually progresses to a secondary progressive type (SPMS) of MS [216]. Immunomodulatory and immunosuppressive drugs are the frontline of current MS treatment that raises the risk of infection and cancer [217]. Alternative diseasemodifying therapies (DMTs) began in the 1990s with interferon- $\beta$ (IFN $\beta$ ) as first-line agents in the treatment of MS [217].

Currently, there are at least six different parenteral formulations FDA-approved MS drugs such as interferons, immunosuppressants, corticosteroids, glatiramer acetate, sphingosine-1-phosphate receptor modulators, and monoclonal antibodies which via targeting immune system at various levels with different mechanisms significantly reduce the frequency and intensity of the attacks in patients with relapsing MS and slow down the progression of the disease [218]. However, contrary to favorable impact of DMT drugs on relapsing MS by preventing the frequency of relapses, they have limited benefit on progressive MS and axonal damage. Also, efficacy, tolerability, and safety of DMT vary between moderate to high levels and even in cases that are very effective, continuing treatment is limited by the risk of serious side effects including cardiomyopathy $[219,220]$. New immunemodulating approaches including stem cell transplantation have emerged in regenerative medicine for the treatment of inflammation-associated diseases. The rationale behind stem cell therapies for MS is loss of oligodendrocytes and myelin sheaths which is the main cause of axonal degeneration and its correlated functional disability [221]. Stem cell therapy in MS is often categorized as an immune reconstitution therapy (IRT) by removing the components of the immune system with the aim of creating an opportunity for self-renewal of the immune system [222]. According to the obtained result by Liu and his colleagues, the main reason of immunomodulatory effects of stem cells is the HLA-G expression, as an inhibitor of natural killer cell (NK) killing, in response to interferon gamma (IFN $\gamma$ ) that is the main inflammatory mediator involved with the onset of MS [223]. This strategy was able to induce long-term or even permanent remission in treatment-free periods of MS. Because the main cause of demyelination of axons in diseases such as MS is autoimmune attacks [224]. Theoretically, tissue repair through stem cell transplantation not only may lead to the reconstruction of axons by replacing destroyed and lost cells but also via paracrine neuroprotective and anti-inflammatory manner which could prevent the progressive axonal and neural degradation [221]. Rajan et al. demonstrated anti-inflammatory and immunosuppressive effects conditioned medium containing exosomes derived from periodontal ligament stem cells in the EAE mouse models of MS. The obtained results show a significant remyelination in the spinal cord due to increase of anti-inflammatory cytokines including IL-10, contrary to decreased level of pro-inflammatory cytokines which eventually slows and reverses the progression of MS [225]. One of the pathological properties of MS is impaired regulatory $\mathrm{T}$ cell function that has a key role in the modulation of Th1/Th2 balance. The clinical benefits of MSC for the modulation of immune responses of autoimmune diseases such as MS depend on their ability to induce $\mathrm{T}$ regulatory responses and switch from Th1 to Th2 [226]. An investigation carried out by Clark and her colleagues have shown that EVs derived from placenta-derived MSCs (PMSCs), in high doses, are able to achieve the clinical benefits of treatment alone in the EAE model. Proteomic analysis revealed the presence of hepatocyte growth factor (HGF) and VEGF in the EVs derived from PMSC. PMSCs through secretion of high levels of these factors modulated the immune system by inducing the regulatory T cell. This result showed that PMSC-EV is able to create immunomodulatory responses comparable to PMSC treatments and induce myelin regeneration in the EAE mouse model [144]. Similarly, intravenous administration of human adipose tissue-derived MSC-EVs improves the score of EAE mice by inhibiting the infiltration of immune cells into the site of injury and suppressing their activation and reducing the production of pro-inflammatory cytokines [134].

As mentioned in the above studies, one of the key aims in the treatment of autoimmunity disease is induction and maintenance of immunological tolerance. One of the recent strategies for peripheral tolerance is maintenance and progresses in the activity of regulatory molecules such as programmed death ligand-1 (PD-L1), galectin-1, and TGF- $\beta$ through biological intervention in host immune system [227]. A study carried out by Mokarizadeh and his colleagues assayed the tolerogenic activities of the content of the MV derived from MSC to induce peripheral tolerance on the splenic mononuclear cells (MNCs) derived from experimental autoimmune encephalomyelitis (EAE)-affected mice. The result demonstrated that MSCs-MV activate the apoptotic signaling in the self-reactive lymphocyte and stimulate them to secrete antiinflammatory cytokines including IL-10 and TGF- $\beta$ and also increase the expression of regulatory molecules such as PDL1 and TGF- $\beta$ on the MV surface which led to inducing the differentiation of regulatory $\mathrm{T}$ cells that induce peripheral tolerance of immune responses [228].

Other emerging strategy for inducing immunological tolerance in patient with MS is polarization of the microglia toward M2 phenotype [229]. Microglia is the resident macrophage within the CNS that under microenvironments is rapidly activated to differentiate into either the M1 which produces proinflammatory cytokines and induces CNS damage or the M2 phenotype that promotes tissue regeneration through production of anti-inflammatory cytokines [230]. Imbalance of M1/ M2 macrophages and switch toward pro-inflammatory M1 phenotype in the early stages of MS have been considered as one of the leading factors to tissue damage in the CNS. Thus, it is believed that stimulating the polarization of microglia toward the M2 phenotype can be effective in the reduction 
of neurological symptoms of MS patients [229]. In this regard, $\mathrm{Li}$ in 2019 evaluated the impact of BMSC paracrine mechanism especially the mediation of exosomes on the microglial polarization and improvement of motor function in the mouse model of EAE [231]. After the comparison of the untreated and treated EAE group, they reported that exosome derived from BMSC by altering the polarization of microglia toward a M2 phenotype can reduce inflammation and demyelination of the CNS and improve the neural behavioral scores in the EAE mouse model [231]. Moreover, they showed that MSC exosome treatment reduced significantly the levels of M1associated interleukin (IL-12) and tumor necrosis factor (TNF)- $\alpha$ while enhancing M2-associated cytokines (TGF- $\beta$ and IL-10) [231]. Another research carried out by Farinazzo linked the decreased activity of CNS immune cells including microglial and $\mathrm{T}$ cell extravasation with reduced demyelination in the spinal cord after treatment by nanovesicles secreted from adipose stem cells (ASC) [232]. Due to exosome's ability to cross the BBB, it can act as a vehicle to transport drugs to the MS patient. In many cases, also, binding different functional groups such as antibodies and aptamers to the surface of the exosomes significantly increase the target specificity of the exosomes. For instance, in 2019, surface functionalized MSC-derived exosomes with anti-myelin aptamer (LJM-3064) have been designed by Shamili et al. to fight against MS. LJM-3064, in addition to the ability to cross the $\mathrm{BBB}$, has high propensity to protein myelin and induction of remyelination which has been confirmed by previous studies. The obtained results showed that co-delivery of LJM-3064 aptamer and MSC exosome reduced symptom severity of illness in the $\mathrm{C} 57 \mathrm{BL} / 6$ mice through both immunomodulation effect of MSC exosome and remyelination effects of LJM3064 aptamer. Actually, in this system, exosome in addition to anti-inflammatory effect acts as a vehicle for the aptamer in improving remyelination effect of LJM-3064 aptamer [145]. According to these results achieved so far, it can be said that the future of MS treatment methods will be based on SCderived exosomes for several strong reasons, including safety, ability to cross the BBB, and their cargo.

\section{Stem Cell-Derived Exosome in Other Neurodegenerative Disease}

Neurodegenerative disease include a wide range of diseases associated with progressive central nervous system damage [233]. In addition to using stem cell-derived exosome-based therapy to treat multiple sclerosis, $\mathrm{AD}$ and $\mathrm{PD}$, this strategy has also been applied to other neurological disorders, such as Huntington's disease (HD), amyotrophic lateral sclerosis (ALS), and traumatic brain injury (TBI) [234].

For instance, Lee et al. demonstrated differentiation of neural stem cell isolated from (SOD1(G93A)) transgenic ALS mice after treatment by ADSC exosomes. The result of the analytical technique showed the reduction of cytosolic superoxide dismutase 1 (SOD1) deposition and alleviation of mitochondrial proteins including phospho-CREB/CREB ratio and PGC-1 $\alpha$ [235]. Similarly, another study confirmed the protective effect of ADMSC-derived exosomes from oxidative damage on the in vitro model expressing ALS mutations [236]. In a study by Rajan in 2017 , human gingival mesenchymal stem cells (hGMSCs) conditioned medium which contain antiinflammatory cytokines such as NGF, NT3, IL-10, and TGF- $\beta$ led to upregulation in the expression of BDNF and neurotrophin-3 (NT-3) that protect motoneuron-like NSC-34 cells against scratch-injury-induced cell death [237].

In HD, a genetic neurodegenerative disease, like other neurodegenerative disease, polyglutamine huntingtin protein is transferred to other cells through the exosome [238]. Therefore, exosomes play an important role in the progression of HD pathology. Several therapy strategy-based exosomes have been evaluated for HD treatment [239]. One study in 2016 by Lee et al. showed that exosomes from ADMSC modulate pathological phenotypes in in vitro model of HD through reduced intracellular aggregate of $\mathrm{mHtt}$, increasing the expression level of PGC-1 and phospho-CREB [240]. Another study by this group demonstrated that miR-124 which is highly decreased in HD patient, delivered to the striatum of the R6/ $2 \mathrm{HD}$ transgenic mice by exosome, despite the decline in the intracellular expression level of REST, as miR-124-target gene, was observed to have little improvement in behavior [241].

The application of stem cell-derived exosomes in neurodegenerative diseases is shown in Table 1. In summary, because different neurodegenerative diseases have similar pathological mechanism, it is more likely that one treatment strategy will work for all of them. Achieving such an effective strategy for different types of diseases requires extensive and comprehensive studies.

\section{Conclusion and Future Perspective}

Nowadays, regeneration and functional recovery of degenerated axons and myelin is considered an ideal option for treating neurodegenerative diseases using stem cell therapy procedures. Self-renewal and differentiation potency of stem cells are their most important properties as medicines for neurodegenerative disease. Although, stem cells remain a valuable therapeutic option for neural regenerative medicine, therapeutic use of these cells is restricted by possible immune reactivity, tumor formation, and inadequate differentiation along with non-specific targeting and lack of capability to cross physiological and biological barriers. Exosomes derived from stem cells not only have therapeutic properties similar their parent cells but also have the advantage of avoiding 
Table 1 The application of stem cell-derived extracellular vesicle in neurodegenerative diseases

\begin{tabular}{ll}
\hline Disease & Origin and type of EVs \\
\hline $\begin{array}{c}\text { Alzheimer's } \\
\text { disease }\end{array}$ & $\begin{array}{c}\text { Bone marrow mesenchymal stem } \\
\text { cells/extracellular vesicles }\end{array}$ \\
& $\begin{array}{c}\text { Bone marrow mesenchymal stem } \\
\text { cells/extracellular vesicles }\end{array}$ \\
& $\begin{array}{c}\text { Human adipose tissue-derived mesenchy- } \\
\text { mal stem cells/extracellular vesicles } \\
\text { Mesenchymal stem cells/exosomes } \\
\text { Cytokine (TNF } \alpha \text { and INF } \gamma \text { ) } \\
\text { preconditioned mesenchymal stem } \\
\text { cells/extracellular vesicles } \\
\text { Wharton's jelly mesenchymal stem } \\
\text { cells/extracellular vesicles }\end{array}$ \\
& \\
Hypoxia-preconditioned mesenchymal \\
stem cells/extracellular vesicles
\end{tabular}
stem cells/extracellular vesicles

Neural stem cells/extracellular vesicles

Parkinson's disease

Heat-shock neural stem cells/exosomes Human exfoliated deciduous teeth stem cells (SHEDs)/exosomes

Human exfoliated deciduous teeth stem cells (SHEDs)/extracellular vesicles

Mesenchymal stem cells/exosomes Mesenchymal stem cells/exosomes

Multiple sclerosis

Periodontal ligament stem cells/exosomes

Placenta-derived MSCs/extracellular vesicles

Mesenchymal stem cells/extracellular vesicles

Not reported

Not reported

Not reported

Intranasal administration

Tail vein injections

Not reported

Intravenous injection

Subcutaneous injections

Mesenchymal stem cells/exosomes

Tail vein injections

Adipose tissue-derived mesenchymal stem Intravenous injections cells/nanovesicles

Human adipose tissue-derived mesenchymal stem cells/extracellular vesicles

Stroke

Bone marrow mesenchymal stem cells/exosomes

Bone marrow mesenchymal stem cells/exosome

Adipose mesenchymal stem cells/extracellular vesicles

Human neural stem cells/extracellular vesicles

Human neural stem cells/extracellular vesicles

Intravenous injection

Intravenous injection

Intravenous injection

Tail vein injection
Outcomes

Ref

Decrease extracellular $\mathrm{A} \beta$ oligomer level through:

- Endocytic and degradation by MSCs

- Secretion EVs containing the catalase

- Release of anti-inflammatory cytokines (IL-6, IL-10, and VEGF)

Prevent $\mathrm{A} \beta$ plaque formation and reduce dystrophic neurons:

- Increase plaque phagocytosis by microglial cells

- Proteolysis of A $\beta$ plaques by neprilysin

Proteolysis of $\mathrm{A} \beta$ plaques by neprilysin

Promote neurogenesis and cognitive function recovery

Improve in dendritic spine density through:

- Downregulation IL-6 and IL-1 $\beta$ and upregulation IL-10

- Polarization microglia toward an anti-inflammatory phenotype

Increases the resistance of hippocampal neurons to damage caused by $\mathrm{A} \beta$ through:

- Regulating the function of astrocytes

- Decreasing ROS production

Reduced intracellular and extracellular deposition of $\mathrm{A} \beta$ oligomers

Ameliorates learning and memory deficits through:

- reduce pro-inflammatory cytokines (IL-1 $\beta$ and TNF- $\alpha$ ) and vice versa, increase inflammatory cytokines (IL-4 and IL-10)

- decrease the activity of STAT3 and NF- $\mathrm{BB}$

Improve cognitive dysfunction through:

- improve mitochondrial function, SIRT1 activation, synaptic activity

- reduction in inflammatory response

Improves cognitive and motor function

Inhibition the apoptosis-induced by (6-OHDA) in human dopaminergic neurons

Improve motor symptoms through:

- normalizes tyrosine hydroxylase expression in the substantia nigra and striatum of the (6-OHDA)-treated rats

Regulate neurite outgrowth by transfer of the miR-133b

Stimulation of oligodendrogenesis and improving neuronal function

Remyelination in the spinal cord through:

- increase of anti-inflammatory cytokines including IL-10 and contrary to decrease the level of pro-inflammatory cytokines

Improving motor function and induce myelin regeneration through:

- modulation immune system and induce the regulatory $\mathrm{T}$ cell differentiation by its growth factors cargo (HGF and VEGF)

Induce peripheral tolerance, active the apoptotic signaling in the self-reactive lymphocyte and induce the differentiation of regulatory $\mathrm{T}$ cells through:

- secretion anti-inflammatory cytokines (IL-10 and TGF- $\beta$ )

-expression of regulatory molecules (PD-L1 and TGF- $\beta$ ) on the MV

Attenuate inflammation and demyelination of the CNS through:

- altering the polarization of microglia toward a M2 phenotype

Reducing demyelination in the spinal cord through:

- decreased activity CNS immune cells including microglial and $\mathrm{T}$ cell

Intravenous injections Attenuates induced-EAE through:

- diminishing proliferative potency of T cells

- leukocyte infiltration

- demyelination on a chronic model of MS

Ameliorates functional recovery and increase axonal density and synaptophysin-positive areas through:

- improves neurite remodeling, neurogenesis, and angiogenesis

Stimulate long-term neuroprotection, promote neuroregeneration and neurological recovery through:

- modulate peripheral post-stroke immune responses

Improve functional recovery through:

- fiber tract integrity, axonal sprouting and white matter repair

Improving behavior and mobility through:

- decrease intracranial hemorrhage in ischemic lesions

- elimination in cerebral lesion volume and decreased brain swelling and reduce edema

[194]

Ameliorate tissue and functional recovery and episodic memory formation through: 
Table 1 (continued)

\begin{tabular}{|c|c|c|c|c|}
\hline Disease & Origin and type of EVs & Route of administration & Outcomes & Ref \\
\hline & $\begin{array}{l}\text { Neural stem cell and human induced } \\
\text { pluripotent stem cell-derived cardio- } \\
\text { myocyte (iCM)/exosome }\end{array}$ & Intravenous injection & $\begin{array}{l}\text { - changing the systemic immune response } \\
\text { Reduced infarct volumes and induce neuroprotection through: } \\
\text { - preservation the function of astrocyte }\end{array}$ & [141] \\
\hline & Mesenchymal stem cells/exosome & Intravenous injection & $\begin{array}{l}\text { Improving behavior function through: } \\
\text { - neurogenesis and angiogenesis mediated by miRNA-184 and } \\
\text { miRNA- } 210\end{array}$ & [142] \\
\hline
\end{tabular}

whole cell post-transplant adverse events due to their ability to pass the physiological barriers, ability to migrate and to reside in the brain lesion sites, high safety profile, and with not yet reported any cases from the immune response and rejection.

In summary, the research to date indicates that stem cellderived exosome-based therapies are the foundation basic for a possible cure in the field of neurodegeneration (Fig. 2). Neuroprotective and neurodegeneration, remyelination, reduction of neural inflammation, and recovery of function after induced injury are some areas that exosome-derived stem cells have plenty of use.

The result of such studies well demonstrates that the development of exosome-based therapy by relying only on the intrinsic properties of the exosome is not possible. As a suggestion, combining the intrinsic properties of stem cell-derived exosomes with a targeted modification could be effective in treating neurodegenerative disorders in the future. Advanced studies have attempted to manipulate and modify the surface or intermolecular contents of stem cell-derived exosomes in order to improve the homing ability and therapy potential whenever necessary for specific purposes. For example, Lamp2-RVG-modified exosomes were confirmed by several investigations to selective delivery of exosomes to the brain after intravenous injection [242, 243]. Lamp2 is an EV membrane-anchored protein fused with RVG glycoprotein that is a neuron-specific peptide [244]. In the same study, Lamp2b was genetically engineered with the $\alpha \mathrm{v}$ integrinspecific iRGD peptide to doxorubicin delivery into $\alpha \mathrm{V}$ integrin-positive tumor tissues [245]. Another study used of engineered exosome constructed from fusion platelet-derived

Fig. 2 The applications of stem cell-derived exosomes in neurodegenerative diseases

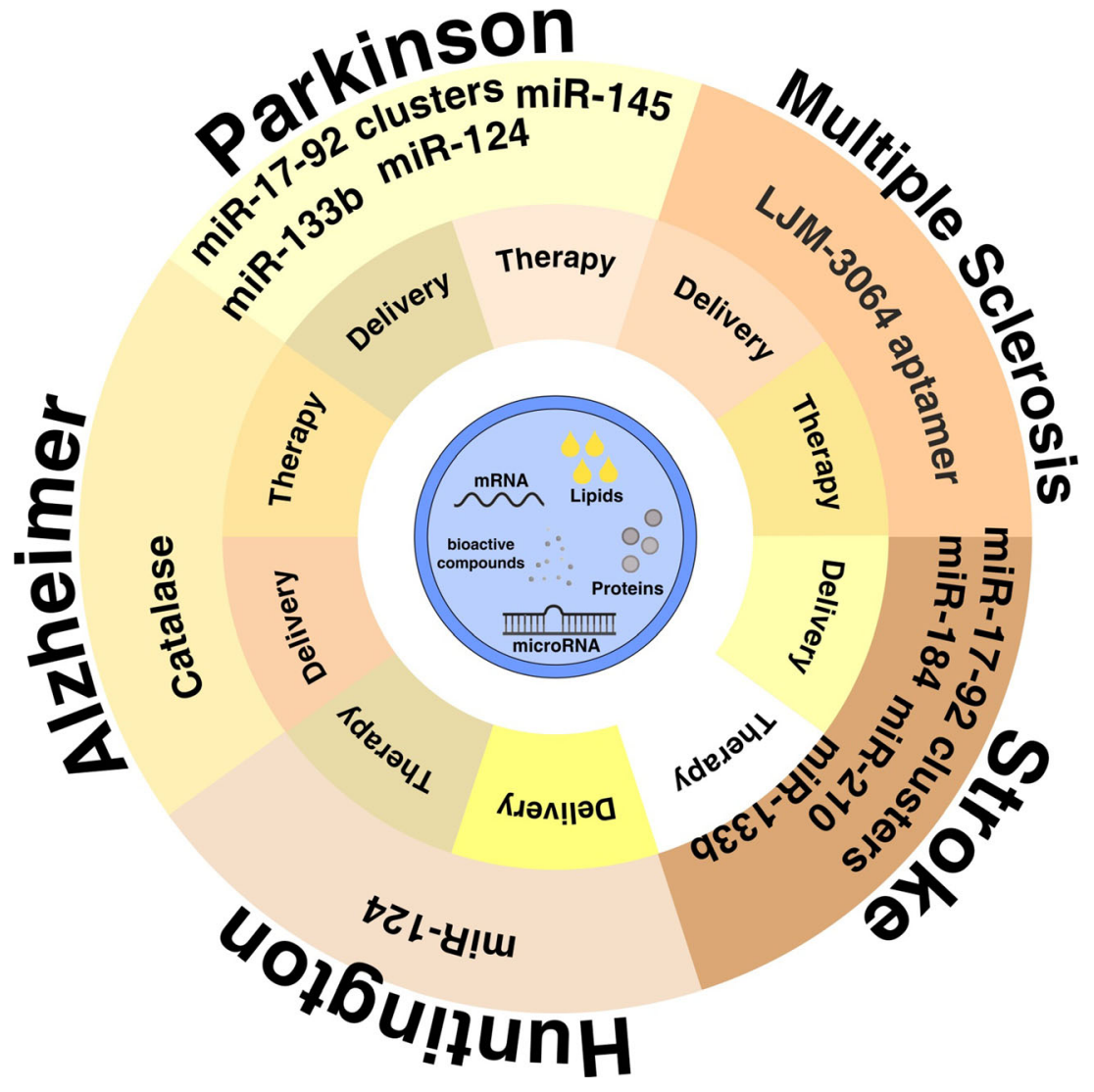


growth factor (PDGF) receptor with GE11 peptide to selective gene delivery to the epidermal growth factor receptor (EGFR) expressing breast cancer mice model [246]. Kooijmans et al. also demonstrated that inhibition of interactions of active groups on the surface exosome with plasma proteins and non-target cells through covering it with polyethylene glycol prolonged their circulation half-life [247]. When referring to the exosomes derived from stem cells, there are some areas including finding the best source of exosome which produced stem cell with neurotherapeutic behavior, identification neuroprotective properties of proteins or miRNA exosome's cargo, and some molecular mechanism such as exosomal cargo selection process and method of taking up exosomes by cells that need further investigations.

Author Contribution All authors contributed to the study conception and design. Rezvan Najafi is responsible for the conception and design of the review. Nashmin Fayazi, Mohsen Sheykhhasan, and Sara Soleimani Asl conducted the literature search and data analysis and drafted the article. All authors reviewed this draft, contributed, and approved the final manuscript.

Data Availability Not applicable.

\section{Declarations}

Ethics Approval This article does not contain any studies involving human participants or animals performed by any of the authors.

Consent to Participate Not applicable.

Consent for Publication All the authors approved the version to be published and agreed to be accountable for all aspects of the work.

Conflict of Interest The authors declare no competing interests.

\section{References}

1. Andreone BJ, Larhammar M, Lewcock JW (2020) Cell death and neurodegeneration. Cold Spring Harb Perspect Biol 12(2): a036434

2. Hou Y, Dan X, Babbar M et al (2019) Ageing as a risk factor for neurodegenerative disease. Nat Rev Neurol 15(10):565-581

3. Agosta F, Galantucci S, Filippi M (2017) Advanced magnetic resonance imaging of neurodegenerative diseases. Neurol Sci 38(1):41-51

4. Erkkinen MG, Kim M-O, Geschwind MD (2018) Clinical neurology and epidemiology of the major neurodegenerative diseases. Cold Spring Harb Perspect Biol 10(4):a033118

5. Chen X, Pan W (2014) The treatment strategies for neurodegenerative diseases by integrative medicine. Integr Med Int 1(4):223225

6. Huang M, Gu X, Gao X (2019) Nanotherapeutic strategies for the treatment of neurodegenerative diseases. Brain targeted drug delivery system. Academic Press, Cambridge, pp 321-356
7. Przedborski S (2017) Neurodegeneration. Neuroimmune pharmacology. T. Ikezu and H. E. Gendelman. Springer International Publishing, Cham, pp 345-354

8. Agrawal M (2020) Molecular basis of chronic neurodegeneration. Clinical Molecular Medicine: Academic Press, Cambridge, pp $447-460$

9. Gandhi J, Antonelli AC, Afridi A et al (2019) Protein misfolding and aggregation in neurodegenerative diseases: a review of pathogeneses, novel detection strategies, and potential therapeutics. Rev Neurosci 30(4):339-358

10. Ramsay RR, Majekova M, Medina M et al (2016) Key targets for multi-target ligands designed to combat neurodegeneration. Front Neurosci 10:375

11. Sankowski R, Mader S, Valdés-Ferrer SI (2015) Systemic inflammation and the brain: novel roles of genetic, molecular, and environmental cues as drivers of neurodegeneration. Front Cell Neurosci 9:28

12. Jankovic J (2016) Dopamine depleters in the treatment of hyperkinetic movement disorders. Expert Opin Pharmacother 17(18): 2461-2470

13. Raschetti R, Albanese E, Vanacore N et al (2007) Cholinesterase inhibitors in mild cognitive impairment: a systematic review of randomised trials. PLoS Med 4(11):e338

14. Chaudhuri KR, Schapira AH (2009) Non-motor symptoms of Parkinson's disease: dopaminergic pathophysiology and treatment. Lancet Neurol 8(5):464-474

15. Umemura A, Jaggi JL, Hurtig HI et al (2003) Deep brain stimulation for movement disorders: morbidity and mortality in 109 patients. J Neurosurg 98(4):779-784

16. Cotzias G, Papavasiliou P, Gellene R (1968) Experimental treatment of parkinsonism with L-DOPA. Neurology. 18(3):276-277

17. Yahr M, Duvoisin R, Hoehn MM et al (1968) L-DOPA (L-3, 4dihydroxyphenylanine)-its clinical effects in parkinsonism. Trans Am Neurol Assoc 93:56-63

18. Comfort A (1978) Cholinesterase inhibition in treatment of Alzheimer's dementia. Lancet 311(8065):659-660

19. Rabins PV, Lyketsos CG (2006) Cholinesterase inhibitors and memantine have a role in the treatment of Alzheimer's disease. Nat Clin Pract Neurol 2(11):578-579

20. Marks WJ Jr, Ostrem JL, Verhagen L et al (2008) Safety and tolerability of intraputaminal delivery of CERE-120 (adeno-associated virus serotype 2-neurturin) to patients with idiopathic Parkinson's disease: an open-label, phase I trial. Lancet Neurol 7(5):400-408

21. Stewart WF, Kawas C, Corrada M et al (1997) Risk of Alzheimer's disease and duration of NSAID use. Neurology. 48(3):626-632

22. Traynor B, Bruijn L, Conwit R et al (2006) Neuroprotective agents for clinical trials in ALS: a systematic assessment. Neurology. 67(1):20-27

23. Ristori G, Romano S, Visconti A et al (2010) Riluzole in cerebellar ataxia: a randomized, double-blind, placebo-controlled pilot trial. Neurology. 74(10):839-845

24. Armstrong, M. J., \& Miyasaki, J. M. (2012). Evidence-based guideline: pharmacologic treatment of chorea in Huntington disease: report of the guideline development subcommittee of the American Academy of Neurology. Neurology 79(6):597-603

25. Sheikh S, Haque E, Mir SS (2013) Neurodegenerative diseases: multifactorial conformational diseases and their therapeutic interventions. J Neurodegener Dis 2013:563481

26. Van der Schyf CJ (2011) The use of multi-target drugs in the treatment of neurodegenerative diseases. Expert Rev Clin Pharmacol 4(3):293-298

27. Smid P, Coolen HK, Keizer HG et al (2005) Synthesis, structure -activity relationships, and biological properties of 1-heteroaryl$4-[\omega-(1 \mathrm{H}$-indol-3-yl) alkyl] piperazines, Novel potential 
antipsychotics combining potent dopamine D2 receptor antagonism with potent serotonin reuptake inhibition. J Med Chem 48(22):6855-6869

28. Morphy R, Rankovic Z (2005) Designed multiple ligands. An emerging drug discovery paradigm. J Med Chem 48(21):65236543

29. Bolognesi ML, Banzi R, Bartolini M et al (2007) Novel class of quinone-bearing polyamines as multi-target-directed ligands to combat Alzheimer's disease. J Med Chem 50(20):4882-4897

30. Morphy R, Rankovic Z (2009) Designing multiple ligandsmedicinal chemistry strategies and challenges. Curr Pharm Des 15(6):587-600

31. Collins PY, Patel V, Joestl SS et al (2011) Grand challenges in global mental health. Nature. 475(7354):27-30

32. Deverman BE, Ravina BM, Bankiewicz KS et al (2018) Gene therapy for neurological disorders: progress and prospects. Nat Rev Drug Discov 17(9):641-659

33. Piguet F, Alves S, Cartier N (2017) Clinical gene therapy for neurodegenerative diseases: past, present, and future. Hum Gene Ther 28(11):988-1003

34. Kolli N, Lu M, Maiti P et al (2018) Application of the gene editing tool, CRISPR-Cas9, for treating neurodegenerative diseases. Neurochem Int 112:187-196

35. Cota A, Diaz NF, Diaz-Martinez E (2019) Editing the central nervous system through CRISPR/Cas9 systems. Front Mol Neurosci 12:110

36. Pahan K (2019) A Broad application of CRISPR Cas9 in infectious, inflammatory and neurodegenerative diseases. J NeuroImmune Pharmacol 14(4):534-536

37. Ghosh R, Tabrizi SJ (2017) Gene suppression approaches to neurodegeneration. Alzheimers Res Ther 9(1):82

38. Harper SQ, Staber PD, He X et al (2005) RNA interference improves motor and neuropathological abnormalities in a Huntington's disease mouse model. Proc Natl Acad Sci 102(16): $5820-5825$

39. JE BAMET (1963) Cytologial demonstration of the clonal nature of spleen colonies derived from transplanted mouse arrow cells. Nature. 197:452-454

40. Chen Y, Liu L (2012) Modern methods for delivery of drugs across the blood-brain barrier. Adv Drug Deliv Rev 64(7):640 665

41. Blanchette M, Fortin D (2011) Blood-brain barrier disruption in the treatment of brain tumors. The blood-brain and other neural barriers 686:447-463. https://doi.org/10.1007/978-1-60761-938$3 \_23$

42. Saraiva C, Praça C, Ferreira R et al (2016) Nanoparticle-mediated brain drug delivery: overcoming blood-brain barrier to treat neurodegenerative diseases. J Control Release 235:34-47

43. Gabathuler R (2010) Approaches to transport therapeutic drugs across the blood-brain barrier to treat brain diseases. Neurobiol Dis 37(1):48-57

44. Drago D, Cossetti C, Iraci N et al (2013) The stem cell secretome and its role in brain repair. Biochimie. 95(12):2271-2285

45. Mirzamohammadi S, Aali E, Najafi R et al (2015) Effect of $17 \beta$ estradiol on mediators involved in mesenchymal stromal cell trafficking in cell therapy of diabetes. Cytotherapy. 17(1):46-57

46. Najafi R, Sharifi AM (2013) Deferoxamine preconditioning potentiates mesenchymal stem cell homing in vitro and in streptozotocin-diabetic rats. Expert Opin Biol Ther 13(7):959-972

47. Pourjafar M, Saidijam M, Mansouri K et al (2017) All-trans retinoic acid preconditioning enhances proliferation, angiogenesis and migration of mesenchymal stem cell in vitro and enhances wound repair in vivo. Cell Prolif 50(1):e12315

48. Kim N, Cho S-G (2013) Clinical applications of mesenchymal stem cells. Korean J Intern Med 28(4):387
49. Biehl JK, Russell B (2009) Introduction to stem cell therapy. J Cardiovasc Nurs 24(2):98-105

50. Yamanaka S (2012) Induced pluripotent stem cells: past, present, and future. Cell Stem Cell 10(6):678-684

51. Fouad GIJBotNRC (2019) Stem cells as a promising therapeutic approach for Alzheimer's disease: a review. Bull Natl Res Cent 43(1):1-20

52. Luzzani CD, Miriuka SG (2017) Pluripotent stem cells as a robust source of mesenchymal stem cells. Stem Cell Rev Rep 13(1):6878

53. Singh VK, Saini A, Kalsan M et al (2016) Describing the stem cell potency: the various methods of functional assessment and in silico diagnostics. Front Cell Dev Biol 4:134-134

54. Condic ML (2014) Totipotency: what it is and what it is not. Stem Cells Dev 23(8):796-812

55. Nie J, Koehler KR, Hashino E (2017) Directed differentiation of mouse embryonic stem cells into inner ear sensory epithelia in 3D culture. Springer, Organ Regeneration, pp. 67-83

56. Elkabetz Y, Studer L (eds.) (2008) Human ESC-derived neural rosettes and neural stem cell progression. Cold Spring Harb Symp Quant Biol 73:377-387. https://doi.org/10.1101/sqb.2008.73.052

57. Volarevic V, Markovic BS, Gazdic M et al (2018) Ethical and safety issues of stem cell-based therapy. Int J Med Sci 15(1):36-45

58. Robinton DA, Daley GQ (2012) The promise of induced pluripotent stem cells in research and therapy. Nature. 481(7381):295305

59. Saito S, Lin Y-C, Nakamura Y et al (2019) Potential application of cell reprogramming techniques for cancer research. Cell Mol Life Sci 76(1):45-65

60. Prentice DA (2019) Adult stem cells: successful standard for regenerative medicine. Circ Res 124(6):837-839

61. He Q, Wan C, Li G (2007) Concise review: multipotent mesenchymal stromal cells in blood. Stem Cells 25(1):69-77

62. Yang H, Feng R, Fu Q et al (2019) Human induced pluripotent stem cell-derived mesenchymal stem cells promote healing via TNF- $\alpha$-stimulated gene- 6 in inflammatory bowel disease models. Cell Death Dis 10(10):1-16

63. Wong S-P, Rowley JE, Redpath AN et al (2015) Pericytes, mesenchymal stem cells and their contributions to tissue repair. Pharmacol Ther 151:107-120

64. Tian X, Brookes O, Battaglia G (2017) Pericytes from mesenchymal stem cells as a model for the blood-brain barrier. Sci Rep 7(1): $1-7$

65. Dore-Duffy P, Katychev A, Wang X et al (2006) CNS microvascular pericytes exhibit multipotential stem cell activity. J Cereb Blood Flow Metab 26(5):613-624

66. Kunisaki Y, Bruns I, Scheiermann C et al (2013) Arteriolar niches maintain haematopoietic stem cell quiescence. Nature. 502(7473): 637-643

67. Yuan X, Wu Q, Wang P et al (2019) Exosomes derived from pericytes improve microcirculation and protect blood-spinal cord barrier after spinal cord injury in mice. Front Neurosci 13:319

68. Kolios G, Moodley Y (2013) Introduction to stem cells and regenerative medicine. Respiration. 85(1):3-10

69. Paul G (2008) Cell transplantation for patients with Parkinson's disease. Stem Cells 174:361-388

70. Lunn JS, Sakowski SA, Hur J et al (2011) Stem cell technology for neurodegenerative diseases. Ann Neurol 70(3):353-361

71. Dantuma E, Merchant S, Sugaya K (2010) Stem cells for the treatment of neurodegenerative diseases. Stem Cell Res Ther 1(5): $1-7$

72. Abshenas R, Artimani T, Amiri I et al (2020) Effects of treadmill exercise and preconditioned bone marrow-derived mesenchymal stem cells transplantation on $\mathrm{A} \beta$-induced neurotoxicity in male rats. Koomesh. 22(2):325-333 
73. Abshenas R, Artimani T, Shahidi S et al (2020) Treadmill exercise enhances the promoting effects of preconditioned stem cells on memory and neurogenesis in $\mathrm{A} \beta$-induced neurotoxicity in the rats. Life Sci 15(249): 117482

74. Cui Y, Ma S, Zhang C et al (2017) Human umbilical cord mesenchymal stem cells transplantation improves cognitive function in Alzheimer's disease mice by decreasing oxidative stress and promoting hippocampal neurogenesis. Behav Brain Res 320:291301

75. Wilkins A, Kemp K, Ginty M et al (2009) Human bone marrowderived mesenchymal stem cells secrete brain-derived neurotrophic factor which promotes neuronal survival in vitro. Stem Cell Res 3(1):63-70

76. Nakano M, Nagaishi K, Konari N et al (2016) Bone marrowderived mesenchymal stem cells improve diabetes-induced cognitive impairment by exosome transfer into damaged neurons and astrocytes. Sci Rep 6:24805

77. Doyle LM, Wang MZ (2019) Overview of extracellular vesicles, their origin, composition, purpose, and methods for exosome isolation and analysis. Cells. 8(7):727

78. Lötvall J, Hill AF, Hochberg F et al (2014) Minimal experimental requirements for definition of extracellular vesicles and their functions: a position statement from the International Society for Extracellular Vesicles. J Extracell Vesicles 22(3):26913. https:// doi.org/10.3402/jev.v3.26913

79. Pap E, Pallinger E, Pasztoi M et al (2009) Highlights of a new type of intercellular communication: microvesicle-based information transfer. Inflamm Res 58(1):1-8

80. Mathivanan S, Simpson RJ (2009) ExoCarta: a compendium of exosomal proteins and RNA. Proteomics. 9(21):4997-5000

81. Szabo G, Momen-Heravi F (2020) Extracellular vesicles and exosomes: biology and pathobiology. The Liver: Biology and Pathobiology. Wiley, New York, pp 1022-1027. https://doi.org/ 10.1002/9781119436812.ch78

82. Antonelou MH, Seghatchian J (2016) Update on extracellular vesicles inside red blood cell storage units: adjust the sails closer to the new wind. Transfus Apher Sci 55(1):92-104

83. Marcus ME, Leonard JN (2013) FedExosomes: engineering therapeutic biological nanoparticles that truly deliver. Pharmaceuticals. 6(5):659-680

84. Witwer KW, Buzás EI, Bemis LT et al (2013) Standardization of sample collection, isolation and analysis methods in extracellular vesicle research. J Extracell Vesic 2(1):20360

85. Batrakova EV, Kim MS (2015) Using exosomes, naturallyequipped nanocarriers, for drug delivery. J Control Release 219: 396-405

86. Muller L (2020) Exosomes: nanodust? HNO. 68(1):56-59

87. Budnik V, Ruiz-Canada C, Wendler F (2016) Extracellular vesicles round off communication in the nervous system. Nat Rev Neurosci 17(3):160-72.10

88. Wiklander OP, Nordin JZ, O'Loughlin A et al (2015) Extracellular vesicle in vivo biodistribution is determined by cell source, route of administration and targeting. J Extracell Vesic 4(1):26316

89. Kalani A, Tyagi A, Tyagi N (2014) Exosomes: mediators of neurodegeneration, neuroprotection and therapeutics. Mol Neurobiol 49(1):590-600

90. Van der Pol E, Coumans F, Grootemaat A et al (2014) Particle size distribution of exosomes and microvesicles determined by transmission electron microscopy, flow cytometry, nanoparticle tracking analysis, and resistive pulse sensing. J Thromb Haemost 12(7): 1182-1192

91. Subra C, Laulagnier K, Perret B et al (2007) Exosome lipidomics unravels lipid sorting at the level of multivesicular bodies. Biochimie. 89(2):205-212
92. Subra C, Grand D, Laulagnier K et al (2010) Exosomes account for vesicle-mediated transcellular transport of activatable phospholipases and prostaglandins. J Lipid Res 51(8):2105-2120

93. Stahl PD, Barbieri MA (2002) Multivesicular bodies and multivesicular endosomes: the "ins and outs" of endosomal traffic. Sci Signal 2002(141):pe32

94. Zöller M (2016) Exosomes in cancer disease. In Cancer Gene Profiling. Humana Press, New York, pp 111-149

95. Gho YS, Lee C (2017) Emergent properties of extracellular vesicles: a holistic approach to decode the complexity of intercellular communication networks. Mol BioSyst 13(7):1291-1296

96. Chen X, Liang H, Zhang J et al (2012) Horizontal transfer of microRNAs: molecular mechanisms and clinical applications. Protein Cell 3(1):28-37

97. Choi DS, Kim DK, Kim YK et al (2013) Proteomics, transcriptomics and lipidomics of exosomes and ectosomes. Proteomics. 13(10-11):1554-1571

98. Llorente A (2020) Mass spectrometry for the identification of protein biomarkers in urinary extracellular vesicles. Proteomic and metabolomic approaches to biomarker discoverypp. Academic Press, pp 437-440

99. Deng F, Miller J (2019) A review on protein markers of exosome from different bio-resources and the antibodies used for characterization. J Histotechnol 42(4):226-239

100. Wortzel I, Dror S, Kenific CM et al (2019) Exosome-mediated metastasis: communication from a distance. Dev Cell 49(3):347360

101. Van Niel G, Porto-Carreiro I, Simoes S et al (2006) Exosomes: a common pathway for a specialized function. J Biochem 140(1): $13-21$

102. Karlsson M, Lundin S, Dahlgren U et al (2001) “Tolerosomes" are produced by intestinal epithelial cells. Eur J Immunol 31(10): 2892-2900

103. Morelli AE, Larregina AT, Shufesky WJ et al (2004) Endocytosis, intracellular sorting, and processing of exosomes by dendritic cells. Blood. 104(10):3257-3266

104. Durak-Kozica M, Baster Z, Kubat K et al (2018) 3D visualization of extracellular vesicle uptake by endothelial cells. Cell Mol Biol Lett 23(1):1-9

105. Huang W (2017) MicroRNAs: biomarkers, diagnostics, and therapeutics. Bioinformatics in microRNA research: Humana Press, New York, pp 57-67

106. Xiao T, Zhang W, Jiao B et al (2017) The role of exosomes in the pathogenesis of Alzheimer' disease. Translat Neurodegener 6(1):3

107. Coleman BM, Hill AF (eds.) (2015) Extracellular vesicles-their role in the packaging and spread of misfolded proteins associated with neurodegenerative diseases. Seminars in Cell \& Developmental Biology. Semin Cell Dev Biol 40:89-96. https:// doi.org/10.1016/j.semcdb.2015.02.007

108. Lugli G, Cohen AM, Bennett DA et al (2015) Plasma exosomal miRNAs in persons with and without Alzheimer disease: altered expression and prospects for biomarkers. PLoS One 10(10): e0139233

109. Quek C, Hill AFJB (2017) The role of extracellular vesicles in neurodegenerative diseases. Commun $\mathrm{Br}$ 483(4):1178-1186

110. Turturici G, Tinnirello R, Sconzo G et al (2014) Extracellular membrane vesicles as a mechanism of cell-to-cell communication: advantages and disadvantages. Am J Phys Cell Phys 306(7): C621-C633

111. Goetzl EJ, Kapogiannis D, Schwartz JB et al (2016) Decreased synaptic proteins in neuronal exosomes of frontotemporal dementia and Alzheimer's disease. FASEB J 30(12):4141-4148

112. Goetzl EJ, Abner EL, Jicha GA et al (2018) Declining levels of functionally specialized synaptic proteins in plasma neuronal exosomes with progression of Alzheimer's disease. FASEB J 32(2):888-893 
113. Mullins RJ, Mustapic M, Goetzl EJ et al (2017) Exosomal biomarkers of brain insulin resistance associated with regional atrophy in Alzheimer's disease. Hum Brain Mapp 38(4):1933-1940

114. Saint-Pol J, Gosselet F, Duban-Deweer S et al (2020) Targeting and crossing the blood-brain barrier with extracellular vesicles. Cells. 9(4):851

115. Gonda A, Kabagwira J, Senthil GN et al (2019) Internalization of exosomes through receptor-mediated endocytosis. Mol Cancer Res 17(2):337-347

116. Chen CC, Liu L, Ma F et al (2016) Elucidation of exosome migration across the blood-brain barrier model in vitro. Cell Mol Bioeng 9(4):509-529

117. Kuroda H, Tachikawa M, Yagi Y et al (2018) Cluster of differentiation 46 Is the major receptor in human blood-brain barrier endothelial cells for uptake of exosomes derived from brainmetastatic melanoma cells (SK-Mel-28). Mol Pharm 16(1):292304

118. Dozio V, Sanchez J-C (2017) Characterisation of extracellular vesicle-subsets derived from brain endothelial cells and analysis of their protein cargo modulation after TNF exposure. J Extracell Vesic 6(1):1302705

119. Solé C, Moliné T, Vidal M et al (2019) An exosomal urinary miRNA signature for early diagnosis of renal fibrosis in lupus nephritis. Cells. 8(8):773

120. Morishita M, Takahashi Y, Nishikawa M et al (2017) Pharmacokinetics of exosomes - an important factor for elucidating the biological roles of exosomes and for the development of exosome-based therapeutics. J Pharm Sci 106(9):2265-2269

121. Koh E, Lee EJ, Nam G-H et al (2017) Exosome-SIRP $\alpha$, a CD47 blockade increases cancer cell phagocytosis. Biomaterials. 121: 121-129

122. Clayton A, Harris CL, Court J et al (2003) Antigen-presenting cell exosomes are protected from complement-mediated lysis by expression of CD55 and CD59. Eur J Immunol 33(2):522-531

123. Kumar S, Michael IJ, Park J et al (2018) Cloaked exosomes: biocompatible, durable, and degradable encapsulation. Small. 14(34): 1802052

124. Zhang Y, Bi J, Huang J et al (2020) Exosome: a review of its classification, isolation techniques, storage, diagnostic and targeted therapy applications. Int J Nanomedicine 15:6917

125. Smyth T, Kullberg M, Malik N et al (2015) Biodistribution and delivery efficiency of unmodified tumor-derived exosomes. J Control Release 199:145-155

126. Munagala R, Aqil F, Jeyabalan J et al (2016) Bovine milk-derived exosomes for drug delivery. Cancer Lett 371(1):48-61

127. Lee C, Mitsialis SA, Aslam M et al (2012) Exosomes mediate the cytoprotective action of mesenchymal stromal cells on hypoxiainduced pulmonary hypertension. Circulation. 126(22):26012611

128. Watson DC, Bayik D, Srivatsan A et al (2016) Efficient production and enhanced tumor delivery of engineered extracellular vesicles. Biomaterials. 105:195-205

129. Hoshino A, Costa-Silva B, Shen T-L et al (2015) Tumour exosome integrins determine organotropic metastasis. Nature. 527(7578):329-335

130. Saunderson SC, Dunn AC, Crocker PR et al (2014) CD169 mediates the capture of exosomes in spleen and lymph node. Blood 123(2):208-216

131. Hall J, Prabhakar S, Balaj L et al (2016) Delivery of therapeutic proteins via extracellular vesicles: review and potential treatments for Parkinson's disease, glioma, and schwannoma. Cell Mol Neurobiol 36(3):417-427

132. Webb RL, Kaiser EE, Scoville SL et al (2018) Human neural stem cell extracellular vesicles improve tissue and functional recovery in the murine thromboembolic stroke model. Stroke 9(5):530-539
133. Chen K-H, Chen C-H, Wallace CG et al (2016) Intravenous administration of xenogenic adipose-derived mesenchymal stem cells (ADMSC) and ADMSC-derived exosomes markedly reduced brain infarct volume and preserved neurological function in rat after acute ischemic stroke. Oncotarget. 7(46):74537

134. Jafarinia M, Alsahebfosoul F, Salehi H et al (2020) Therapeutic effects of extracellular vesicles from human adipose-derived mesenchymal stem cells on chronic experimental autoimmune encephalomyelitis. J Cell Physiol 235(11):8779-8790

135. Perets N, Betzer O, Shapira R et al (2019) Golden exosomes selectively target brain pathologies in neurodegenerative and neurodevelopmental disorders. Nano Lett 19(6):3422-3431

136. Reza-Zaldivar EE, Hernández-Sapiéns MA, Gutiérrez-Mercado YK et al (2019) Mesenchymal stem cell-derived exosomes promote neurogenesis and cognitive function recovery in a mouse model of Alzheimer's disease. Neural Regen Res 14(9):1626

137. Liu Y, Zheng Y, Karatas H et al (2017) 12/15-Lipoxygenase inhibition or knockout reduces warfarin-associated hemorrhagic transformation after experimental stroke. Stroke. 48(2):445-451

138. Xin H, Li Y, Liu Z et al (2013) MiR-133b promotes neural plasticity and functional recovery after treatment of stroke with multipotent mesenchymal stromal cells in rats via transfer of exosome-enriched extracellular particles. Stem Cells 31(12): $2737-2746$

139. Doeppner TR, Herz J, Görgens A et al (2015) Extracellular vesicles improve post-stroke neuroregeneration and prevent postischemic immunosuppression. Stem Cells Transl Med 4(10): $1131-1143$

140. Xin H, Wang F, Li Y et al (2017) Secondary release of exosomes from astrocytes contributes to the increase in neural plasticity and improvement of functional recovery after stroke in rats treated with exosomes harvested from microRNA 133b-overexpressing multipotent mesenchymal stromal cells. Cell Transplant 26(2): 243-257

141. Sun X, Jung JH, Arvola OJ et al (2019) Stem cell-derived exosomes protect astrocyte cultures from in vitro ischemia and decrease injury as post-stroke intravenous therapy. Front Cell Neurosci 13:394

142. Moon GJ, Sung JH, Kim DH et al (2019) Application of mesenchymal stem cell-derived extracellular vesicles for stroke: biodistribution and microRNA study. Transl Stroke Res 10(5): 509-521

143. Go V, Bowley BG, Pessina MA et al (2020) Extracellular vesicles from mesenchymal stem cells reduce microglial-mediated neuroinflammation after cortical injury in aged rhesus monkeys. Geroscience. 42(1):1-17

144. Clark K, Zhang S, Barthe S et al (2019) Placental mesenchymal stem cell-derived extracellular vesicles promote myelin regeneration in an animal model of multiple sclerosis. Cells. 8(12):1497

145. Shamili FH, Alibolandi M, Rafatpanah H et al (2019) Immunomodulatory properties of MSC-derived exosomes armed with high affinity aptamer toward mylein as a platform for reducing multiple sclerosis clinical score. J Control Release 299:149_ 164

146. Elia CA, Tamborini M, Rasile M et al (2019) Intracerebral injection of extracellular vesicles from mesenchymal stem cells exerts reduced $A \beta$ plaque burden in early stages of a preclinical model of Alzheimer's disease. Cells 8(9):1059

147. Losurdo M, Pedrazzoli M, D'Agostino C et al (2020) Intranasal delivery of mesenchymal stem cell-derived extracellular vesicles exerts immunomodulatory and neuroprotective effects in a $3 \times \mathrm{Tg}$ model of Alzheimer's disease. Stem Cells Transl Med 9(9):10681084

148. Li B, Liu J, Gu G et al (2020) Impact of neural stem cell-derived extracellular vesicles on mitochondrial dysfunction, sirtuin 1 level, 
and synaptic deficits in Alzheimer's disease. J Neurochem 154(5): 502-518

149. Ophelders DR, Wolfs TG, Jellema RK et al (2016) Mesenchymal stromal cell-derived extracellular vesicles protect the fetal brain after hypoxia-ischemia. Stem Cells Transl Med 5(6):754-763

150. Drommelschmidt K, Serdar M, Bendix I et al (2017) Mesenchymal stem cell-derived extracellular vesicles ameliorate inflammation-induced preterm brain injury. Brain Behav Immun 60:220-232

151. Chen P, Zheng L, Wang Y et al (2019) Desktop-stereolithography 3D printing of a radially oriented extracellular matrix/ mesenchymal stem cell exosome bioink for osteochondral defect regeneration. Theranostics 9(9):2439

152. Narbute K, Pilipenko V, Pupure J et al (2019) Intranasal administration of extracellular vesicles derived from human teeth stem cells improves motor symptoms and normalizes tyrosine hydroxylase expression in the substantia nigra and striatum of the 6hydroxydopamine-treated rats. Stem Cells Transl Med 8(5):490499

153. Huber C (2020) Utilizing neural stem cell-derived exosomes for the treatment of Alzheimer's disease. IdeaFest 36. https://red. library.usd.edu/idea/36

154. Otero-Ortega L, Laso-García F, del Carmen Gómez-de Frutos M et al (2017) White matter repair after extracellular vesicles administration in an experimental animal model of subcortical stroke. Sci Rep 7(1):1-11

155. Lackland DT, Roccella EJ, Deutsch AF et al (2014) Factors influencing the decline in stroke mortality: a statement from the American Heart Association/American Stroke Association. Stroke 45(1):315-353

156. Coull A, Lovett J, Rothwell P (2004) Population based study of early risk of stroke after transient ischaemic attack or minor stroke: implications for public education and organisation of services. BMJ. 328(7435):326

157. Jivan K, Ranchod K, Modi G (2013) Management of ischaemic stroke in the acute setting: review of the current status. Cardiovasc J Africa 24(3):88

158. Cheng NT, Kim AS (2015) Intravenous thrombolysis for acute ischemic stroke within 3 hours versus between 3 and 4.5 hours of symptom onset. Neurohospitalist 5(3):101-109

159. Boyle K, Joundi RA, Aviv RI (2017) An historical and contemporary review of endovascular therapy for acute ischemic stroke. Neurovasc Imaging 3(1):1

160. Kapral MK, Wang H, Mamdani M et al (2002) Effect of socioeconomic status on treatment and mortality after stroke. STROKE-DALLAS-. 33(1):268-273

161. Mendis S (2013) Stroke disability and rehabilitation of stroke: World Health Organization perspective. Int J Stroke 8(1):3-4

162. Xin H, Li Y, Cui Y et al (2013) Systemic administration of exosomes released from mesenchymal stromal cells promote functional recovery and neurovascular plasticity after stroke in rats. J Cereb Blood Flow Metab 33(11):1711-1715

163. Webb RL, Kaiser EE, Jurgielewicz BJ et al (2018) Human neural stem cell extracellular vesicles improve recovery in a porcine model of ischemic stroke. Stroke 49(5):1248-1256

164. Ullah M, Ng NN, Concepcion W et al (2020) Emerging role of stem cell-derived extracellular microRNAs in age-associated human diseases and in different therapies of longevity. Ageing Res Rev 57:100979

165. Xin H, Katakowski M, Wang F et al (2017) MicroRNA-17-92 cluster in exosomes enhance neuroplasticity and functional recovery after stroke in rats. Stroke. 48(3):747-753

166. Barzegar M, Wang Y, Yun JW et al (2020) Abstract TP102: human placental mesenchymal stem cells derived exosomeangiotensin converting enzyme-2 dependent protection in ischemic stroke injury. Stroke (Vol. 51). Two Commerce SQ, 2001
Market St, Philadelpia, PA 19103 USA: Lippincott Williams \& Wilkins

167. Wang C, Börger V, Sardari M et al (2020) Mesenchymal stromal cell-derived small extracellular vesicles induce ischemic neuroprotection by modulating leukocytes and specifically neutrophils. Stroke 51(6):1825-1834

168. Deng Y, Chen D, Gao F et al (2019) Exosomes derived from microRNA-138-5p-overexpressing bone marrow-derived mesenchymal stem cells confer neuroprotection to astrocytes following ischemic stroke via inhibition of LCN2. J Biol Eng 13:71. https:// doi.org/10.1186/s13036-019-0193-0

169. Safakheil M, Safakheil HJ (2020) The effect of exosomes derived from bone marrow stem cells in combination with rosuvastatin on functional recovery and neuroprotection in rats after ischemic stroke. J Mol Neurosci 70(5):724-737

170. Xia Y, Ling X, Hu G et al (2020) Small extracellular vesicles secreted by human iPSC-derived MSC enhance angiogenesis through inhibiting STAT3-dependent autophagy in ischemic stroke. Stem Cell Res Ther 22;11(1):313. https://doi.org/10. 1186/s13287-020-01834-0

171. Venkat P, Zacharek A, Landschoot-Ward J et al (2020) Exosomes derived from bone marrow mesenchymal stem cells harvested from type two diabetes rats promotes neurorestorative effects after stroke in type two diabetes rats. Exp Neurol 334:113456. https:// doi.org/10.1016/j.expneurol.2020.113456

172. Reiss AB, Arain HA, Stecker MM et al (2018) Amyloid toxicity in Alzheimer's disease. Rev Neurosci 29(6):613-627

173. De Strooper B, Karran E (2016) The cellular phase of Alzheimer's disease. Cell. 164(4):603-615

174. Tai H-C, Wang BY, Serrano-Pozo A et al (2014) Frequent and symmetric deposition of misfolded tau oligomers within presynaptic and postsynaptic terminals in Alzheimer's disease. Acta Neuropathol Commun 2(1):146

175. Du X, Wang X, Geng M (2018) Alzheimer's disease hypothesis and related therapies. Translat Neurodegener 7(1):2

176. Birks JS, Harvey RJ (2018) Donepezil for dementia due to Alzheimer's disease. Cochrane Database of Systematic Reviews, Issue 6. Art. No.: CD001190. https://doi.org/10.1002/14651858. CD001190.pub3

177. Takahashi RH, Nagao T, Gouras GK (2017) Plaque formation and the intraneuronal accumulation of $\beta$-amyloid in Alzheimer's disease. Pathol Int 67(4):185-193

178. Small SA, Simoes-Spassov S, Mayeux R et al (2017) Endosomal traffic jams represent a pathogenic hub and therapeutic target in Alzheimer's disease. Trends Neurosci 40(10):592-602

179. Hamlett ED, Goetzl EJ, Ledreux A et al (2017) Neuronal exosomes reveal Alzheimer's disease biomarkers in Down syndrome. Alzheimers Dement 13(5):541-549

180. Pusic KM, Grinberg YY, Kraig RP et al (2019) Exosome-based therapeutics against neurodegenerative disorders. U.S. Patent Application 16/259,563

181. Abner EL, Elahi FM, Jicha GA et al (2020) Endothelial-derived plasma exosome proteins in Alzheimer's disease angiopathy. FASEB J 34(4):5967-5974

182. Saman S, Kim W, Raya M et al (2012) Exosome-associated tau is secreted in tauopathy models and is selectively phosphorylated in cerebrospinal fluid in early Alzheimer disease. J Biol Chem 287(6):3842-3849

183. Lewczuk P, Kornhuber J, Vanmechelen E et al (2010) Amyloid $\beta$ peptides in plasma in early diagnosis of Alzheimer's disease: a multicenter study with multiplexing. Exp Neurol 223(2):366-370

184. Fiandaca MS, Kapogiannis D, Mapstone M et al (2015) Identification of preclinical Alzheimer's disease by a profile of pathogenic proteins in neurally derived blood exosomes: a casecontrol study. Alzheimers Dement 11(6):600-607. e1 
185. Clark CM, Xie S, Chittams J et al (2003) Cerebrospinal fluid tau and $\beta$-amyloid: how well do these biomarkers reflect autopsyconfirmed dementia diagnoses? Arch Neurol 60(12):1696-1702

186. Cheng Á, Doecke JD, Sharples R et al (2015) Prognostic serum miRNA biomarkers associated with Alzheimer's disease shows concordance with neuropsychological and neuroimaging assessment. Mol Psychiatry 20(10):1188-1196

187. Gui Y, Liu H, Zhang L et al (2015) Altered microRNA profiles in cerebrospinal fluid exosome in Parkinson disease and Alzheimer disease. Oncotarget. 6(35):37043

188. Riancho J, Vázquez-Higuera JL, Pozueta A et al (2017) MicroRNA profile in patients with Alzheimer's disease: analysis of miR-9-5p and miR-598 in raw and exosome enriched cerebrospinal fluid samples. J Alzheimers Dis 57(2):483-491

189. Mattsson N, Zetterberg H, Janelidze S et al (2016) Plasma tau in Alzheimer disease. Neurology. 87(17):1827-1835

190. Wang S, Cesca F, Loers G et al (2011) Synapsin I is an oligomannose-carrying glycoprotein, acts as an oligomannosebinding lectin, and promotes neurite outgrowth and neuronal survival when released via glia-derived exosomes. J Neurosci 31(20):7275-7290

191. Yuyama K, Sun H, Mitsutake S et al (2012) Sphingolipidmodulated exosome secretion promotes clearance of amyloid- $\beta$ by microglia. J Biol Chem 287(14):10977-10989

192. Yuyama K, Sun H, Sakai S et al (2014) Decreased amyloid- $\beta$ pathologies by intracerebral loading of glycosphingolipidenriched exosomes in Alzheimer model mice. J Biol Chem 289(35):24488-24498

193. Katsuda T, Oki K, Ochiya T (2014) Potential application of extracellular vesicles of human adipose tissue-derived mesenchymal stem cells in Alzheimer's disease therapeutics. Stem cell renewal and cell-cell communication: Humana Press, New York, pp 171181

194. de Godoy MA, Saraiva LM, de Carvalho LR et al (2018) Mesenchymal stem cells and cell-derived extracellular vesicles protect hippocampal neurons from oxidative stress and synapse damage induced by amyloid- $\beta$ oligomers. J Biol Chem 293(6): 1957-1975

195. Bodart-Santos V, de Carvalho LR, de Godoy MA et al (2019) Extracellular vesicles derived from human Wharton's jelly mesenchymal stem cells protect hippocampal neurons from oxidative stress and synapse damage induced by amyloid- $\beta$ oligomers. Stem Cell Res Ther 10(1):1-13

196. Cui G-H, Wu J, Mou F-F et al (2018) Exosomes derived from hypoxia-preconditioned mesenchymal stromal cells ameliorate cognitive decline by rescuing synaptic dysfunction and regulating inflammatory responses in APP/PS1 mice. FASEB J 32(2):654 668

197. Pringsheim T, Jette N, Frolkis A et al (2014) The prevalence of Parkinson's disease: a systematic review and meta-analysis. Mov Disord 29(13):1583-1590

198. Yu H, Sun T, An J et al (2020) Potential roles of exosomes in Parkinson's disease: from pathogenesis, diagnosis, and treatment to prognosis. Front Cell Dev Biol 8:86

199. Mendes Filho D (2018) dC Ribeiro P, Oliveira LF, et al. Therapy with mesenchymal stem cells in Parkinson disease: history and perspectives. Neurologist 23(4):141-147

200. Hornykiewicz O (2015) 50 years of levodopa. Mov Disord 30(7): 1008-1008

201. Ascherio A, Schwarzschild MA (2016) The epidemiology of Parkinson's disease: risk factors and prevention. Lancet Neurol 15(12):1257-1272

202. Chang Y-H, Wu K-C, Harn H-J et al (2018) Exosomes and stem cells in degenerative disease diagnosis and therapy. Cell Transplant 27(3):349-363
203. Teixeira FG, Carvalho MM, Panchalingam KM et al (2017) Impact of the secretome of human mesenchymal stem cells on brain structure and animal behavior in a rat model of Parkinson's disease. Stem Cells Transl Med 6(2):634-646

204. Pinheiro BM, Anjo SI, Manadas B et al (2019) Bone marrow mesenchymal stem cells' secretome exerts neuroprotective effects in a Parkinson's disease rat model. Front Bioeng Biotechnol 7:294

205. Oh SH, Kim HN, Park HJ et al (2017) The cleavage effect of mesenchymal stem cell and its derived matrix metalloproteinase2 on extracellular $\alpha$-synuclein aggregates in Parkinsonian models. Stem Cells Transl Med 6(3):949-961

206. Jarmalavičiūtė A, Tunaitis V, Pivoraitè U et al (2015) Exosomes from dental pulp stem cells rescue human dopaminergic neurons from 6-hydroxy-dopamine-induced apoptosis. Cytotherapy. 17(7):932-939

207. Leggio L, Vivarelli S, L'Episcopo F et al (2017) microRNAs in Parkinson's disease: from pathogenesis to novel diagnostic and therapeutic approaches. Int J Mol Sci 18(12):2698

208. Sonntag K-C (2010) MicroRNAs and deregulated gene expression networks in neurodegeneration. Brain Res 1338:48-57

209. Miñones-Moyano E, Porta S, Escaramís G et al (2011) MicroRNA profiling of Parkinson's disease brains identifies early downregulation of miR-34b/c which modulate mitochondrial function. Hum Mol Genet 20(15):3067-3078

210. Chen Y, Gao C, Sun Q et al (2017) MicroRNA-4639 is a regulator of DJ-1 expression and a potential early diagnostic marker for Parkinson's disease. Front Aging Neurosci 9:232

211. Lee HK, Finniss S, Cazacu S et al (2014) Mesenchymal stem cells deliver exogenous miRNAs to neural cells and induce their differentiation and glutamate transporter expression. Stem Cells Dev 23(23):2851-2861

212. Xin H, Li Y, Buller B et al (2012) Exosome-mediated transfer of miR-133b from multipotent mesenchymal stromal cells to neural cells contributes to neurite outgrowth. Stem Cells 30(7):15561564

213. Dutta R, Trapp BD (2011) Mechanisms of neuronal dysfunction and degeneration in multiple sclerosis. Prog Neurobiol 93(1):1-12

214. Trapp BD, Bö L, Mörk S et al (1999) Pathogenesis of tissue injury in MS lesions. J Neuroimmunol 98(1):49-56

215. Criste G, Trapp B, Dutta R (2014) Axonal loss in multiple sclerosis: causes and mechanisms. Handb Clin Neurol 1(122):101-113

216. Weis S, Sonnberger M, Dunzinger A et al (2019) Demyelinating diseases: multiple sclerosis. imaging brain diseases. Springer, Vienna, pp 1071-1095

217. Panitch HS, Hirsch RL, Schindler J et al (1987) Treatment of multiple sclerosis with gamma interferon: exacerbations associated with activation of the immune system. Neurology. 37(7):10971097

218. Vosoughi R, Freedman MS (2010) Therapy of MS. Clin Neurol Neurosurg 112(5):365-385

219. Rizvi SA, Stone JA, Chaudhry ST et al (2020) Clinical decisionmaking in the management of multiple sclerosis. Clinical neuroimmunology: Springer, pp 159-177

220. Gross RH, Corboy JR (2019) Monitoring, switching, and stopping multiple sclerosis disease-modifying therapies. CONTINUUM: Lifelong Learn Neurol 25(3):715-735

221. Lopez-Diego RS, Weiner HL (2008) Novel therapeutic strategies for multiple sclerosis - a multifaceted adversary. Nat Rev Drug Discov 7(11):909-925

222. Sorensen PS, Sellebjerg F (2019) Pulsed immune reconstitution therapy in multiple sclerosis. Ther Adv Neurol Disord 28(12):112. https://doi.org/10.1177/1756286419836913

223. Liu K-J, Wang C-J, Chang C-J et al (2011) Surface expression of HLA-G is involved in mediating immunomodulatory effects of placenta-derived multipotent cells (PDMCs) towards natural killer lymphocytes. Cell Transplant 20(11-12):1721-1730 
224. Yim H, Jeong H, Oh I (2019) Efficacy and safety of mesenchymal stem cell therapies for patients with multiple sclerosis: a systematic review and single arm meta-analysis. Cytotherapy. 21(5):S50

225. Rajan TS, Giacoppo S, Diomede F et al (2016) The secretome of periodontal ligament stem cells from MS patients protects against EAE. Sci Rep 6:38743

226. Yang H, Sun J, Wang F et al (2016) Umbilical cord-derived mesenchymal stem cells reversed the suppressive deficiency of T regulatory cells from peripheral blood of patients with multiple sclerosis in a co-culture-a preliminary study. Oncotarget. 7(45):72537

227. Shahbaz S, Bozorgmehr N, Koleva P et al (2018) CD71+ VISTA+ erythroid cells promote the development and function of regulatory T cells through TGF- $\beta$. PLoS Biol 16(12):e2006649

228. Mokarizadeh A, Delirezh N, Morshedi A et al (2012) Microvesicles derived from mesenchymal stem cells: potent organelles for induction of tolerogenic signaling. Immunol Lett 147(1-2):47-54

229. Chu F, Shi M, Zheng C et al (2018) The roles of macrophages and microglia in multiple sclerosis and experimental autoimmune encephalomyelitis. J Neuroimmunol 318:1-7

230. Song GJ, Suk K (2017) Pharmacological modulation of functional phenotypes of microglia in neurodegenerative diseases. Front Aging Neurosci 9:139

231. Li Z, Liu F, He X et al (2019) Exosomes derived from mesenchymal stem cells attenuate inflammation and demyelination of the central nervous system in EAE rats by regulating the polarization of microglia. Int Immunopharmacol 67:268-280

232. Farinazzo A, Angiari S, Turano E et al (2018) Nanovesicles from adipose-derived mesenchymal stem cells inhibit $\mathrm{T}$ lymphocyte trafficking and ameliorate chronic experimental autoimmune encephalomyelitis. Sci Rep 8(1):1-11

233. Gitler AD, Dhillon P, Shorter J (2017) Neurodegenerative disease: models, mechanisms, and a new hope. Dis Model Mech 1;10(5): 499-502

234. Magistretti PJ, Geisler FH, Schneider J et al (2019) Gangliosides: treatment avenues in neurodegenerative disease. Front Neurol 10: 859

235. Lee M, Ban J-J, Kim KY et al (2016) Adipose-derived stem cell exosomes alleviate pathology of amyotrophic lateral sclerosis in vitro. Biochem Biophys Res Commun 479(3):434-439
236. Bonafede R, Scambi I, Peroni D et al (2016) Exosome derived from murine adipose-derived stromal cells: neuroprotective effect on in vitro model of amyotrophic lateral sclerosis. Exp Cell Res 340(1):150-158

237. Rajan TS, Diomede F, Bramanti P et al (2017) Conditioned medium from human gingival mesenchymal stem cells protects motor-neuron-like NSC-34 cells against scratch-injury-induced cell death. Int J Immunopathol Pharmacol 30(4):383-394

238. Caron NS, Wright GE, Hayden MR (2018) Huntington disease. GeneReviews ${ }^{\circledR}$ [Internet]. University of Washington, Seattle

239. Croese T, Furlan R (2018) Extracellular vesicles in neurodegenerative diseases. Mol Asp Med 60:52-61

240. Lee M, Liu T, Im W et al (2016) Exosomes from adipose-derived stem cells ameliorate phenotype of Huntington's disease in vitro model. Eur J Neurosci 44(4):2114-2119

241. Lee S-T, Im W, Ban J-J et al (2017) Exosome-based delivery of miR-124 in a Huntington's disease model. J Movement Disord 10(1):45

242. Chivero ET, Liao K, Niu F et al (2020) Engineered extracellular vesicles loaded with miR-124 attenuate cocaine-mediated activation of microglia. Front Cell Dev Biol 8:573

243. Cooper JM, Wiklander PO, Nordin JZ et al (2014) Systemic exosomal siRNA delivery reduced alpha-synuclein aggregates in brains of transgenic mice. Mov Disord 29(12):1476-1485

244. Sokolov A, Kostin N, Ovchinnikova L et al (2019) Targeted drug delivery in lipid-like nanocages and extracellular vesicles. Acta Naturae (англоязычная версия) 11(2):41

245. Tian Y, Li S, Song J et al (2014) A doxorubicin delivery platform using engineered natural membrane vesicle exosomes for targeted tumor therapy. Biomaterials. 35(7):2383-2390

246. S-i O, Takanashi M, Sudo K et al (2013) Systemically injected exosomes targeted to EGFR deliver antitumor microRNA to breast cancer cells. Mol Ther 21(1):185-191

247. Kooijmans S, Fliervoet L, Van Der Meel R et al (2016) PEGylated and targeted extracellular vesicles display enhanced cell specificity and circulation time. J Control Release 224:77-85

Publisher's Note Springer Nature remains neutral with regard to jurisdictional claims in published maps and institutional affiliations. 